(c) 2018, American Psychological Association. This paper is not the copy of record and may not exactly replicate the final, authoritative version of the article. Please do not copy or cite without authors permission. The final article will be available, upon publication, via its DOI: 10.1037/met0000191

\title{
Semantic Measures: Using Natural Language Processing to Measure, Differentiate and Describe Psychological Constructs
}

\author{
Oscar N.E. Kjell, ${ }^{1 *}$ Katarina Kjell, ${ }^{1}$ Danilo Garcia, ${ }^{2,3}$, Sverker Sikström ${ }^{1 *}$
}

\begin{abstract}
Affiliations:
${ }^{1}$ Department of Psychology, Lund University, Lund, Sweden.

${ }^{2}$ Blekinge Center for Competence, Blekinge County Council, Karlskrona, Sweden.

${ }^{3}$ Department of Psychology, University of Gothenburg, Gothenburg, Sweden.

*Correspondence to: Oscar.Kjell@psy.lu.se and Sverker.Sikstrom@psy.lu.se
\end{abstract}

\begin{abstract}
Author Notes: Correspondence and requests for material should be addressed to O.N.E.K. (oscar.kjell@ psy.lu.se) and S.S. (sverker.sikstrom@psy.lu.se). Parts of this paper were presented at the 31st International Congress of Psychology in Yokohama, Japan, July 24-29, 2016. This research is supported by a grant from the Swedish Research Council (2015-01229). O.N.E.K. and S.S. conceived the studies on reports regarding external stimuli and subjective states of harmony and satisfaction; K.K., O.N.E.K. and S.S. conceived the studies on reports regarding subjective states of depression and worry. O.N.E.K. and K.K. collected the data. O.N.E.K., K.K. and S.S. analyzed the data. S.S. developed the software for analyzing the parts using natural language processing. O.N.E.K., K.K., D.G. and S.S. wrote the manuscript. The authors have no competing interest to declare.
\end{abstract}




\section{Abstract}

Psychological constructs, such as emotions, thoughts and attitudes are often measured by asking individuals to reply to questions using closed-ended numerical rating scales. However, when asking people about their state of mind in a natural context ("How are you?"), we receive open-ended answers using words ("fine and happy!") and not closed-ended answers using numbers ("7”) or categories (“A lot"). Nevertheless, to date it has been difficult to objectively quantify responses to open-ended questions. We develop an approach using open-ended questions in which the responses are analyzed using natural language processing (Latent Semantic Analyses). This approach of using open-ended, semantic questions is compared with traditional rating scales in nine studies ( $\mathrm{N}=92-854)$, including two different study paradigms. The first paradigm requires participants to describe psychological aspects of external stimuli (facial expressions) and the second paradigm involves asking participants to report their subjective well-being and mental health problems. The results demonstrate that the approach using semantic questions yields good statistical properties with competitive, or higher, validity and reliability compared with corresponding numerical rating scales. As these semantic measures are based on natural language and measure, differentiate and describe psychological constructs, they have the potential of complementing and extending traditional rating scales.

Keywords: Psychological Assessment; Natural Language Processing; Latent Semantic Analyses. 


\section{Non-Technical Abstract}

We develop tools called semantic measures to statistically measure, differentiate and describe subjective psychological states. In this new method, natural language processing is used for objectively quantifying words from open-ended questions, rather than the closed-ended numerical rating scales traditionally used today. Importantly, the results suggest that these semantic measures have competitive, or higher, validity and reliability compared with traditional rating scales. Using semantic measures also brings along advantages, including an empirical description/definition of the measured construct and better differentiation between similar constructs. This method encompasses a great potential in terms of improving the way we quantify and understand individuals' states of mind. Semantic measures may end up becoming a widespread alternative applied in scientific research (e.g., psychology and medicine) as well as in various professional contexts (e.g., political polls and job recruitment). 
Rating scales is the dominant method used for measuring people's mental states, and is widely used in behavioral and social sciences, but also in practical applications. These scales consist of items such as I am satisfied with my life (Diener, Emmons, Larsen \& Griffin, 1985) coupled with predefined response formats, often ranging from $1=$ strongly disagree to $7=$ strongly agree (Likert, 1932). Hence, this method requires one-dimensional, closed-ended answers from respondents. These methods furthermore require the participants to perform the cognitive task of translating their mental states, or natural language responses, into the one-dimensional response format to make them fit current methods in behavioral sciences. In contrast, we argue that future methods in the field should be adapted to the response format used by people, where natural language processing and machine learning may solve the problem of translating language into scales. In summary, the burden of translating mental states into scientific measureable units should be placed on the method, not the respondents. Furthermore, this method conveys limited information concerning respondents' states of mind, as their options for expressing themselves are limited. For example, an individual answering " 7 " here indicates a high level of satisfaction, but there is no information as to how the respondent has interpreted the item or upon which aspects he or she based the answer: Did the individual consider his or her financial situation, relationships with others, both or perhaps something entirely different?

Although numerical rating scales are widespread, easily quantifiable and have resulted in important findings in different fields, they have drawbacks (e.g., see Krosnick, 1999), which are addressed by our approach. Taking advantage of the human ability to communicate using natural language, we propose a method for enabling open-ended responses that are statistically analyzed by means of semantic measures. We argue that this method contains several advantages over numerical rating scales. First, in daily life subjective states tend to be communicated with words rather than numbers. A person wanting to find out how their friend feels or thinks tends to allow open-ended answers rather than requiring closed-ended 
numerically rated responses. However, the rating scale method requires the respondent (rather than the experimental or statistical methods) to perform the mapping of their natural language responses into a one-dimensional scale. Second, the respondent is not forced to answer using (rather arbitrary) numerical rating scales, but is encouraged to provide reflective, open answers. Third, the construct measured is immediately interpreted by respondents, allowing them to freely elaborate on a personally fitting answer. Closed-ended items use a fixed response format imposed by the test developer (e.g., Kjell, 2011), whereas semantic questions (e.g., "Overall in your life, are you satisfied or not?") allow respondents to answer freely concerning what they perceive to be important aspects of a psychological construct. Finally, the semantic measures may also describe the to-be-measured construct; for example, by revealing statistically significant keywords describing or defining various dimensions in focus. In sum, it could be argued that verbal responses have a higher ecological and face validity compared with rating scales.

There has been a lack of methods for quantifying language (i.e., mapping words to numbers) to capture self-reported psychological constructs. However, computational methods have been introduced in social sciences as a method for quantifying language (e.g., Neuman \& Cohen, 2014; Kern et al., 2016; Park et al., 2014; Pennebaker, Mehl \& Niederhoffer, 2003). A commonly used automated text analysis approach within psychology is the word frequency procedure by Pennebaker et al. (e.g., 2001) referred to as Linguistic Inquiry and Word Count (LIWC). Word frequency approaches are based on hand-coded dictionaries, where human judges have arranged words into different categories, such as Pronouns, Articles, Positive emotions, Negative emotions, Friends, etc. (Pennebaker, Boyd, Jordan \& Blackburn, 2015). Thus, the results from a LIWC analysis reveal the percentages of words in a text categorized in accordance with these predefined categories. This technique has been successful in examining how individuals use the language (e.g., their writing style), but less successful in 
examining the content of what is being said (Pennebaker et al., 2003). Pennebaker et al. (2003) point out that content-based dictionaries encounter problems dealing with all the possible topics people discuss, thus leading to poorer results due to difficulties in terms of categorizing all of these possible topics.

LIWC also suffers from other drawbacks. Categorizations of words as either belonging or not belonging to a category does not reflect the fact that words are more or less prototypical of a category. For example, words such as neutral, concerned and depressed differ in negative emotional tone, whereas LIWC would require each word to either belong or not belong to a negative emotion category. LIWC also fails to capture complex nuances between words. For example, in LIWC2015 (Pennebaker et al., 2015) love and nice both belong to the positive emotion category, which fails to represent differences in, for example, their valence and arousal. Hence, this binary method is limited in terms of capturing nuances in language and complex interrelationships between words. A more precise measure should acknowledge the degree of semantic similarity between words. Pennebaker et al. (2003) conclude that "the most promising content or theme-based approaches to text analysis involve word pattern analyses such as LSA [Latent Semantic Analysis]" (p. 571). LSA allows researchers to automatically map numbers to words within a language.

Sikström has recently developed a web-based program called Semantic Excel (www.semanticexcel.com), which performs different natural language processing analyses (Garcia \& Sikström, 2013; Gustafsson Sendén, Sikström \& Lindholm, 2015; Kjell, Daukantaitė, Hefferon \& Sikström, 2015; Roll et al., 2012). To quantify texts, Semantic Excel is currently based on a method similar to that of LSA, which is both a theory and a method for acquiring and representing the meaning of words (Landauer, 1999; Landauer \& Dumais, 1997; Landauer, Foltz \& Laham, 1998; Landauer, McNamara, Dennis \& Kintsch, 2007). 
LSA assumes that the contexts in which a specific word appears convey information about the meaning of that word. Hence, the first step in LSA involves representing text as a matrix of word co-occurrence counts, where the rows represent words and the columns represent a text passage or some other kind of context (e.g., Landauer, Foltz \& Laham, 1998). Singular value decomposition (Golub \& Kahan, 1965) is applied to this matrix to reduce the dimensionality, and this high-dimensional structure is referred to as a semantic space. Ultimately, each word is represented by an ordered set of numbers (a vector), referred to as a semantic representation. These numbers may be seen as coordinates describing how the words relate to each other in a high-dimensional semantic space. The closer the "coordinates" of two words are positioned within the space, the more similar they are in terms of meaning.

LSA has performed well in several text processing tasks, such as determining whether the contents of various texts have similar meaning (Foltz, Kintsch \& Landauer, 1998). Further, McNamara (2011) points out that LSA has inspired the development of new methods for producing semantic representations, such as Correlated Occurrence Analog to Lexical Semantics (COALS; Rohde, Gonnerman \& Plaut, 2005). We believe that semantic representations from other similar methods would also work for our proposed method using semantic measures. However, importantly the LSA approach has recently been successfully applied within psychology for predicting psychological constructs from texts (Arvidsson, Sikström \& Werbart, 2011; Garcia \& Sikström, 2013; Karlsson, Sikström \& Willander, 2013; Kjell et al., 2015; Kwantes, Derbentseva, Lam, Vartanian \& Marmurek, 2016; Roll et al., 2012). Nevertheless, these predictions are typically performed on free texts written for very different purposes, such as status updates on Facebook, autobiographical memories, description of parents and essays answering hypothetical scenarios. Hence, they are not collected with the objective of efficiently measuring a specific construct. They are thus not 
tailored for complementing rating scales, which probably is the most dominant approach to collect self-report data in behavioral science to date.

To the best of our knowledge, natural language processing has not been applied as a method for complementing and extending rating scales. Compared with LIWC's binary categorizations of words, LSA provides a finer and continues measure of semantic similarity, which reflects the nuances between semantic concepts. We believe that LSA's multidimensional representation of the meaning of words is particularly suitable for measuring psychological constructs. Further, we argue that the multidimensional information included in this natural language processing method may complement and extend the one-dimensional response formats of rating scales. This is why we aim to adapt and further develop these LSAbased computational methods in order to create a semantic measures method capable of both measuring and describing a targeted construct based on open-ended responses to a semantic question. This method has the potential of statistically capturing how individuals naturally answer questions about any type of psychological construct.

\section{Methodological and Statistical Approach}

The various semantic representations and analyses used in this article are described next, and descriptions of key terms relating to the developed method are presented in Table 1. The first part describes the semantic space and its semantic representations. This includes how semantic representations are added to describe several words and how artefacts relating to frequently occurring words are controlled for. The second part describes how the semantic representations are used for different analytical procedures in the forthcoming studies. This includes testing the relationship between words and a numerical variable (i.e., semantic-numeric correlations), predicting properties such as the valence of words/text (i.e., semantic predictions), measuring the semantic similarity between two words/texts (i.e., semantic similarities) and testing whether 
two sets of words/texts statistically differ (i.e., semantic t-tests). Semantic Excel was used for all included studies, as it is capable of both producing and analyzing semantic representations.

Table 1.

Brief Description of Key Terms Relating to Semantic Measures

\begin{tabular}{ll}
\hline Term & Description \\
\hline Semantic measures & $\begin{array}{l}\text { umbrella term for measures based on semantic representations. } \\
\text { a question/item developed to produce responses appropriate for analyses } \\
\text { of semantic representations. } \\
\text { the response to a semantic question (e.g., descriptive words). } \\
\text { Semantic word/text response } \\
\text { Word-norm }\end{array}$ \\
$\begin{array}{l}\text { a collection of words representing a particular understanding of a } \\
\text { construct; a high word-norm refers to the construct under investigation } \\
\text { (e.g., happy) and optionally a low word-norm refers to the construct's }\end{array}$ \\
$\begin{array}{l}\text { opposite meaning (e.g., not at all happy). } \\
\text { a matrix (here based on LSA) in which words (in rows) are described on } \\
\text { several dimensions (in columns) that represent how all words relate to } \\
\text { each other. } \\
\text { the vector (i.e., an ordered set of numerical values) that words are } \\
\text { assigned from the semantic space. } \\
\text { is the value specifying the semantic similarity between two words/texts, } \\
\text { derived by calculating the cosine of the angle between the semantic } \\
\text { representations of each word/text. } \\
\text { the semantic similarity between semantic responses and a high (or low) } \\
\text { word norm. } \\
\text { the semantic similarity of the high norm minus the similarity similarity } \\
\text { of the low norm. } \\
\text { the relationship between the semantic representations of words and a } \\
\text { numeric variable such as a rating scale. } \\
\text { the predicted/estimated property of a word/text such as valence. } \\
\text { the statistical test of whether two sets of words/texts differ in meaning. }\end{array}$ \\
Unipolar scale & $\begin{array}{l}\text { Bipolar scale } \\
\text { Semantic-numeric correlation }\end{array}$ \\
Semantic prediction &
\end{tabular}

\section{The Semantic Space and its Semantic Representations}

The Semantic Space. An approach similar to LSA as implemented by Landauer and Dumais (1997) is employed to produce the semantic space and its semantic representations. Creating high-quality semantic representations requires a very large dataset, much larger than the data collected within the current studies. Therefore, a semantic space was created to function as a "model" for the smaller datasets generated in the current studies. Whereas some researchers produce domain-specific semantic spaces (e.g., if diaries are studied, the semantic space is based on a large amount of text from other diaries), we instead use a general semantic space. Although this might to some extent decrease the domain-specific semantic precision of 
semantic representations, it does make different studies more comparable with each other, while also simplifying analyses.

The current semantic space was originally created using a massive amount of text data $\left(1.7 \times 10^{9}\right.$ words) summarized in the English (version 20120701) Google N-gram database (https://books.google.com/ngrams). LSA typically uses document contexts (Landuaer \& Dumais, 1997), whereas COALS uses a (ramped) "window" of four words (i.e., the four closest words on both sides of a target word, where the closest words have the highest weight; Rohde, Gonnerman \& Plaut, 2005). Rohde, Gonnerman and Plaut (2005) found that smaller text corpora require larger windows, whereas for larger text corpora smaller window sizes are adequate without sacrificing performance. Since our corpus may be considered very large, we use 5-gram rather than documents. Using 5-gram contexts from the database, a co-occurrence (word by word) matrix was set up, where the rows contained the 120k most common words in the n-gram database and the columns consisted of the 10k most common words in the n-gram database $^{1}$. Hence, each cell in the co-occurrence matrix denoted the frequency at which the words in the associated row/column co-occur within the 5-gram.

To increase/decrease the importance of infrequent and frequent words, log-frequency was used; meaning that the cells were normalized by computing the natural logarithm plus one (for different weighting functions, see for instance Nakov, Popova \& Mateev, 2001). Singular value decomposition was then used for compressing the matrix (whilst at the same time preserving as much information as possible). This was carried out to keep the most informative data whilst leaving out "noise". To identify how many dimensions to use in the space, some kind of a synonym test is typically conducted. Landauer and Dumais (1997) use a relatively short synonym test taken from a Test of English as a Foreign Language (TOEFL); however,

\footnotetext{
${ }^{1}$ Although some remove the most common words such as she, he, the and $a$, we keep them, since some of these words may be of interest (e.g., see Gustafsson Sendén, Lindholm, \& Sikström, 2014) and valid results can be achieved even when keeping them.
} 
we applied a more extensive test. This test analyzed how closely synonym word pairs from a thesaurus are positioned to each other in relation to the other words in the semantic space. Thus, the quality of the semantic space is measured by the rank order of the number of words positioned closer to one of the two synonym words than the two words are positioned to each other. The fewer words that are positioned closer to any of the synonym words than the synonyms themselves, the better quality is attributed to the semantic space. Testing the sequence of the powers of 2 (i.e., $1,2,4, \ldots 256,512,1024)$ dimensions, we found that the best test score was achieved using 512 dimensions, which was subsequently considered the optimal number of dimensions. This semantic space has been demonstrated to be valid in previous research (e.g., Kjell et al., 2015).

The Semantic Representation of Participant Responses. By adding the semantic representations of single words (whilst normalizing the length of the vector to one), one may capture and summarize the meaning of several words and paragraphs. Hence, the words generated by participants were assigned their semantic representations from the semantic space; and then all the words' representations for an answer were being summed up and normalized to the length of one. However, the word/text responses generated by the participants were first cleaned using a manual procedure assisted with the spelling tool in Microsoft Words, so that words were spelled according to American spelling. Misspelled words were corrected when the meaning was clear or were otherwise ignored. Instances of successively repeated words or where participants had written "N/A" or the like were excluded. Answers including more than one word in response boxes only requiring one descriptive word were also excluded.

Controlling for Artifacts Relating to Frequently Occurring Words. When aggregating the words to a semantic representation, a normalization is conducted to correct for artifacts related to word frequency. This is achieved by first calculating a frequency-weighted (taken 
from Google N-gram) average of all semantic representations in the space ( $\left.x_{\text {mean }}\right)$, so that the weighting is proportional to how frequently the words occur in Google N-gram. This representation is then subtracted prior to aggregating each word and then added to the final value (i.e., Normalization $\left.\left(\sum_{i=1}^{N}\left(x_{i}-x_{\text {mean }}\right)\right)+x_{\text {mean }}\right)$, where $N$ is the number of words in the text used for creating the semantic representation, and Normalization is a function normalizing the length of the resulting vector to one.

\section{Using the Semantic Representations in Analyses}

Semantic-numeric correlations. The semantic representations can be used for analyzing the relationship between semantic responses and a numerical variable (e.g., numerical rating scale scores). This is achieved by first translating the semantic responses into corresponding semantic representations (as described above), followed by predicting the corresponding numeric rating scales on the basis of these representations by means of multiple linear regression analyses; i.e., $y=\beta_{0}+\beta_{1} * x_{1} \ldots \beta_{m} * x_{m}+\varepsilon$, where $\mathrm{y}$ is the to-be predicted numerical value, $\beta_{0}$ is the intercept, $x_{1}$ through $x_{m}$ are the predictors (i.e., the values from the m number of semantic representations; $x_{1}=$ the first dimension of the semantic representations, $x_{2}$ the second dimension and so on) and $\beta_{1}$ through $\beta_{m}$ are the coefficients defining the relationship between the semantic dimensions and the rating scale scores. When the predicted variable is categorical, multinomial logistic regression is used.

To avoid overfitting the model not all semantic dimensions are used. The number of semantic dimensions to be included in the analysis is here optimized by selecting the first dimensions that best predict the actual score as evaluated using a leave-10\%-out crossvalidation procedure. More specifically, the optimization involves testing different numbers of dimensions, starting with the first dimension(s), which carries most of the information and adding more until all dimensions have been tested. The set of dimensions that best predict the outcome variable is finally used. The sequence used for adding more semantic dimensions aims 
to initially increase a few dimensions each time and then gradually increase by larger numbers. In practice, this was simply achieved by adding 1 , then multiplying by 1.3 and finally rounding to the nearest integer (e.g., 1, 3, 5, 8, where the next number of dimensions to be tested are the first 12; in other words $(8+1) * 1.3)$. In previous research, we have found this sequence to be valid and computationally efficient (e.g., see Kjell et al., 2016; Sarwar, Sikström, Allwood, \& Innes-Ker, 2015). Subsequently, by using leave-10\%-out cross-validation, the validity of the created semantic predicted scales may be tested by assessing its correlation to the numeric variable.

Semantic predictions. To further understand the relationship between words and numerical variables, it is often helpful to estimate various semantic-psychological dimensions of words such as valence. The semantic representations may be used for estimating these dimensions based on independently trained models. This approach uses previously trained semantic models and applies them to new data. In the current studies, we use the Affective Norms for English Words (ANEW; Bradley \& Lang, 1999) to create a semantic trained model for valence. ANEW is a pre-existing comprehensive list of words where participants have rated the words according to several dimensions, such as valence (ranging from unpleasant to pleasant). This enabled us to train each word in the word list to its specific valence rating with a semantic-numeric correlation of $r=.72(p<.001, \mathrm{~N}=1031)$. This trained model of valence is then applied to participants' answers to the semantic questions in order to create a semantic predicted scale of ANEW valence.

Semantic Similarity Scales. To measure the level of similarity between a semantic response and a psychological construct, we compute the semantic similarity between semantic responses and word norms, which describes the endpoints of the psychological dimensions. For example, a high semantic similarity between the answer to a semantic question regarding harmony in life and the word norm for harmony suggests a high level of harmony in life (see 
Figure 1). These word norms are developed by an independent sample of participants generating words describing their view of the constructs being studied (high norms, e.g., "harmony in life") and their opposite meaning (low norms, e.g., "disharmony in life"). The semantic representations of the word norms are generated from participants' replies to word norm questions, where the semantic representations from all the generated words are summed up and the resulting vector is normalized to the length of one.

The semantic similarity between two vectors is calculated as the cosines of the angle. Since the vectors have been normalized to the length of one, this calculation is the same as the dot product (i.e., $\sum_{i=1}^{m} a_{i} b_{i}=a_{1} b_{1}+a_{2} b_{2}+\cdots+a_{m} b_{m}$, where $a$ and $b$ refers to the two semantic representations, $\Sigma$ refers to summation and $\mathrm{m}$ is the number of semantic dimensions). These semantic similarity scales between the semantic representations of semantic responses and word norms are either unipolar (i.e., similarity to the high norm of the to-be-measured construct) or bipolar (i.e. high minus low similarity values).

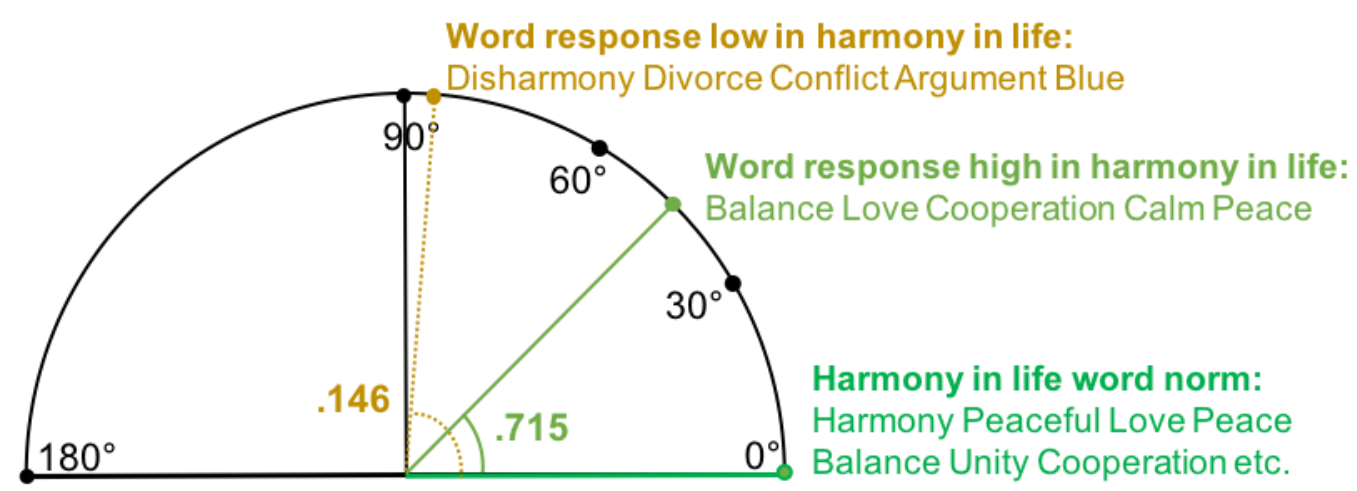

Figure 1. A conceptual illustration of the semantic similarities between word responses and a word norm. The word response high in harmony in life is positioned closer to the harmony in life word norm than the response low in harmony in life. Decimal numbers represent cosine, where the word response high in harmony in life yields a higher cosine (i.e., semantic similarity) than the word response low in harmony in life. 
Semantic T-Test. The difference between two sets of texts may be tested using the semantic representations (c.f. Arvidsson et al., 2011). This is achieved by first creating a semantic representation reflecting the semantic difference between the two sets of texts; we refer to this vector as a semantic difference representation. The semantic similarity is then measured between this semantic difference representation and each individual semantic response. Finally, these semantic similarity values of the two sets of texts may be compared by means of a $t$-test. However, to avoid biasing the results, these steps also include a leave-10\%out procedure.

This is specifically carried out by leaving-out $10 \%$ of the responses before adding the semantic representations for each of the two sets of texts to be compared. Then one of the two semantic representations is subtracted from the other to create the semantic difference representation. The semantic similarity is computed between the semantic difference representation and the semantic representations of each semantic response initially left out in the leave-10\%-out procedure. The leave-10\%-out procedure is repeated until all responses have been used to produce a semantic similarity score. Finally, the difference in semantic similarity between the two sets of texts is tested using a standard $t$-test in order to attain a $p$-value and an effect size.

In two different methodological paradigms, we empirically examine the validity and reliability of semantic measures in relation to traditional numeric rating scales. First, we develop semantic measures to describe external stimuli and examine whether these measures more accurately categorize facial expressions and yield higher inter-rater reliability compared with numerical rating scales. Second, we develop semantic measures for subjective states and examine the validity and reliability of subjective reports related to mental health. In all analyses, alpha was set at .05. 


\section{Reports regarding External Stimuli}

Studies 1 and 2 focused on reports regarding external stimuli, where participants made judgments based on facial expressions, and reports regarding different aspects of the stimuli. The term external is here used to emphasize that all participants describe (their subjective interpretation of) identical facial expressions rather than, for example, describing their subjective states (which is the focus in Study 3-9). We tested whether the semantic measures were capable of categorizing and describing facial expressions from a validated picture database, including happy, sad and contemptuous (Langner et al., 2010). The pictures of facial expressions were selected from a validation study in which human models were trained and coached by specialists to reliably express highly prototypical and recognizable facial expressions (Langner et al., 2010). Hence, these validated facial expressions were seen as the gold standard in relation to categorizing the responses as correct or not. The semantic questions required participants to describe the expressions using three descriptive words. This was compared with the methodological design validating the database (Langner et al., 2010), where expressions were categorized using checkboxes or rated using 5-point scales covering the following dimensions: degree of happiness, sadness and contempt, as well as valence, arousal, intensity, clarity and genuineness.

The pictures were selected based on the highest inter-rater agreement of the targeted facial expression (Langner et al., 2010). Our studies included pictures depicting neutral, happy, sad and contemptuous expressions. Neutral expressions were used for ensuring that the design remained the same as the original validation study, in which participants first evaluated the attractiveness of each model. Happy and sad were selected to align with the forthcoming part on subjective reports regarding mental health. Contemptuous was selected as it has been shown in previous studies that this expression is difficult to label, and thus had the lowest inter-rater reliability (Langner et al., 2010). This difficulty demonstrates a suitable challenge for the 
semantic questions approach, where it may potentially provide information on how participants perceive and describe this expression. Semantic questions enable participants to openly describe their perception of the face, rather than asking participants to tick a checkbox (Study 1) or use a labeled rating scale (Study 2). Hence, semantic questions are proposed to yield a higher level of accuracy when categorizing pictures as well as higher inter-rater reliability.

\section{Methods}

\section{Participants}

Participants were recruited using Mechanical Turk (www.mturk.com), which is an online platform that enables offering payment to individuals to partake in studies. Mechanical Turk as a means of collecting research data within psychology has demonstrated results similar to more conventional methods, as well as good generalizability (Buhrmester, Kwang \& Gosling, 2011). The samples for the studies concerning external stimuli are described in Table 2. 
Table 2

Information about Participants within each Study for Objective Stimuli

\begin{tabular}{|c|c|c|c|c|c|c|}
\hline Study: Condition & $\begin{array}{l}\mathbf{N} \\
\text { (Excluded } \\
\text { due to } \\
\text { control } \\
\text { questions) }^{1}\end{array}$ & $\begin{array}{l}\text { Age } \\
\text { Min- } \\
\text { Max; } \\
\text { Mean } \\
\text { (SD) } \\
\text { years }\end{array}$ & Gender & Nationality & $\begin{array}{l}\text { Mean time } \\
\text { (SD) in } \\
\text { minutes } \\
\text { and } \\
\text { seconds }\end{array}$ & $\begin{array}{c}\text { Payment } \\
\text { in US\$ }\end{array}$ \\
\hline $\begin{array}{l}\text { Study 1: Rating } \\
\text { scales condition }\end{array}$ & 148 & $\begin{array}{l}18-68 \\
34.45 \\
(13.04)\end{array}$ & $\begin{array}{l}\mathrm{F}=56.8 \% \\
\mathrm{M}=42.6 \% \\
\mathrm{O}=0.7 \%\end{array}$ & $\begin{array}{l}\text { US }=87.2 \% \\
\mathrm{IN}=10.8 \% \\
\mathrm{O}=2 \%\end{array}$ & $9.32(5.47)$ & 0.50 \\
\hline $\begin{array}{l}\text { Study 1: } \\
\text { Semantic } \\
\text { questions } \\
\text { condition }\end{array}$ & 119 & $\begin{array}{l}20-65 \\
35.70 \\
(11.28)\end{array}$ & $\begin{array}{l}\mathrm{F}=58.8 \% \\
\mathrm{M}=40.3 \% \\
\mathrm{O}=0.8 \%\end{array}$ & $\begin{array}{l}\mathrm{US}=89.1 \% \\
\mathrm{IN}=6.7 \% \\
\mathrm{O}=4.2 \%\end{array}$ & $14.38(8.31)$ & 0.50 \\
\hline $\begin{array}{l}\text { Study 2: Rating } \\
\text { scales condition }\end{array}$ & $183(7)$ & $\begin{array}{l}19-74 ; \\
34.25 \\
(11.67)\end{array}$ & $\begin{array}{l}\mathrm{F}=54.7 \% \\
\mathrm{M}=44.8 \% \\
\mathrm{O}=0.6 \%\end{array}$ & $\begin{array}{l}\mathrm{US}=80.7 \% \\
\mathrm{IN}=17.1 \% \\
\mathrm{O}=2.2 \%\end{array}$ & $11.23(8.08)$ & 0.50 \\
\hline $\begin{array}{l}\text { Study 2: } \\
\text { Semantic } \\
\text { questions } \\
\text { condition }\end{array}$ & $134(5)$ & $\begin{array}{l}19-67 ; \\
34.03 \\
(11.57)\end{array}$ & $\begin{array}{l}\mathrm{F}=63.2 \% \\
\mathrm{M}=36.1 \\
\mathrm{O}=0.8\end{array}$ & $\begin{array}{l}\mathrm{US}=85 \% \\
\mathrm{IN}=12.8 \% \\
\mathrm{O}=2.3 \%\end{array}$ & $13.44(8.20)$ & 0.50 \\
\hline \multicolumn{7}{|l|}{ Word-Norms } \\
\hline $\begin{array}{l}\text { Face Norms: } \\
\text { Expressions }\end{array}$ & $107(3)$ & $\begin{array}{l}19-61 \\
32.22 \\
(9.54)\end{array}$ & $\begin{array}{l}\mathrm{F}=51.4 \% \\
\mathrm{M}=47.7 \% \\
\mathrm{O}=0.9 \%\end{array}$ & $\begin{array}{l}\mathrm{US}=95.3 \% \\
\mathrm{IN}=3.7 \% \\
\mathrm{O}=0.9 \%\end{array}$ & $6.56(4.59)$ & 0.40 \\
\hline $\begin{array}{l}\text { Face Norms: } \\
\text { Dimensions }\end{array}$ & $107(3)$ & $\begin{array}{l}20-64 \\
33.97 \\
(10.20)\end{array}$ & $\begin{array}{l}\mathrm{F}=62.6 \% \\
\mathrm{M}=37.4 \%\end{array}$ & $\begin{array}{l}\mathrm{US}=81.3 \% \\
\mathrm{IN}=15.9 \% \\
\mathrm{O}=2.8 \%\end{array}$ & $\begin{array}{l}19.22 \\
(14.02)\end{array}$ & 0.40 \\
\hline
\end{tabular}

Note. $\mathrm{F}=$ Female; $\mathrm{M}=$ Male; $\mathrm{O}=$ Other; $\mathrm{US}=$ United States of America; IN=India

${ }^{1}$ In Study 1 there were no control questions.

\section{Instruments and Material}

Pictures of facial expressions from the Radboud Faces Database (Langner et al., 2010) were used. Study 1 and 2 included pictures of six face models displaying four different facial expressions, including happy, sad, contemptuous and neutral.

Rating scales for external stimuli included the instruction to: "Please look at the picture below. Answer the questions about how you interpret the expression." As in the validation study (Langner et al., 2010), Study 1 included nine checkbox alternatives describing different expressions (i.e., "happy," “sad," “contemptuous," "angry," “disgusted," "fearful," "surprised," "neutral" and "other"); as well as 5-point scales for the intensity ("weak" to 
"strong"), the clarity ("unclear" to "clear"), the genuineness ("fake" to "genuine") and the valence ("negative" to "positive") of the expressions. In Study 2, the nine alternatives were replaced by three rating scales concerning to what extent the three expressions "happy," "sad" and "contemptuous" were expressed in the pictures ( $1=$ "not at all" to $5=$ "very much"). This condition also included a rating scale for degree of arousal $(1=$ "low" to $5=$ "high"). With regard to the neutral pictures, only the rating scale for attractiveness was presented.

Semantic items for external stimuli first included the following general instructions: "Please look at the pictures. Answer the question about how you interpret the expressions. Please answer with one descriptive word in each box." The instructions were followed by the following semantic item: "Describe the expression in the picture with three descriptive words." For the neutral pictures in Study 1, the attractiveness question was phrased: "How attractive do you think the person in the picture is? Please answer with one descriptive word in each box." In Study 2, the first part was further clarified to read: "How unattractive or attractive do you think the person in the picture is?" Three boxes were presented underneath each question.

Word norms for external stimuli were generated by asking participants to imagine an expression. This was carried out to attain a general norm, which is not dependent on specific pictures depicting an expression. The following instructions were used: "Please imagine that you should describe the expression of a face in a picture. Write 5 words that best describe a facial expression of happy and 5 words that best describe a facial expression of not at all happy. Please only write one word in each box." These instructions were adapted for "sad" and "contemptuous," as well as to cover the rating scales used in the validation study of the pictures, including: valence (i.e., negative versus positive), arousal (i.e., low versus high), intensity (i.e., weak versus strong), clarity (i.e., unclear versus clear), genuineness (i.e., fake versus genuine) and attractiveness (i.e., unattractive versus attractive). The targeted word (e.g., happy for the 
happy norm) was added to the norm with a frequency of one word more than the most frequently generated word by the participants (i.e., $\mathrm{f}(\max )+1$; see discussion in SM).

\section{Procedure}

All studies were carried out using the following general structure. First, participants were informed about the survey, confidentiality, their right to withdraw at any time without giving a reason and that they could contact the research leader with any questions about the survey. They were asked to agree to the consent form and subsequently complete the survey. Last, demographic information was collected and participants were presented with debriefing information.

In both Study 1 and 2, participants evaluated the various faces from the Radboud Faces Database. The studies involved two conditions: semantic questions and rating scales. Participants were randomly assigned to one of the conditions within the survey. The semantic questions condition was the same for Study 1 and 2, in which the semantic questions and instructions were presented with each of the 24 pictures. In both studies, participants started evaluating the randomly presented neutral pictures in relation to their attractiveness, followed by the randomly presented pictures depicting the different expressions. In the rating scales condition of Study 1, the same design as in the validation study (Langner et al., 2010) was used in which the nine facial expression alternatives were presented, whereas the three rating scales were presented in Study 2. The word norms relating to the reports on external stimuli were collected in two separate studies; one for the expressions and another for the dimensions. The word norm questions were presented in random order.

\section{Results}

The detailed results for Study 1 are presented in the Supplementary Material (SM). Descriptive data of the number of words generated by participants in the semantic questions is presented in Table S1 in SM. In Study 2, the semantic questions and the rating scales conditions both 
involved a $1 / 3$ chance of being correct through random categorization. The semantic responses produced $83.1 \%$ correct categorizations of facial expressions when using semantic predicted scales. This was achieved by training the semantic representations to the expressed facial expression using semantic predicted scales based on multinomial logistic regressions where participants were used as the grouping variable. When grouping the training to pictures, the overall level of correctness reached $78.8 \%$. In comparison, the rating scales responses produced an overall correct categorization (i.e., the targeted expression receiving the highest rating score) of $74.2 \%$. Hence, grouping according to participants $\left(\chi^{2}(1)=62.95, p<.001\right)$ or pictures $\left(\chi^{2}(1)=15.75, p<.001\right)$ yields significantly higher levels of overall correct categorizations compared with using rating scales.

It is also possible to categorize the facial expressions using word norms. The semantic similarity scores between the responses to each picture and the word norms was normalized to z-scores. A response was categorized as correct when the semantic similarity with the word norm representing the facial expression depicted in the picture was the highest. When using semantic similarity scales, the overall correct categorization reached $80.7 \%$ with unipolar scales (i.e., happy, sad and contemptuous) and $80.1 \%$ with bipolar scales (i.e., subtracting "not at all happy," "not at all sad" and "not at all contemptuous" for each construct respectively). Hence, both unipolar and bipolar scales yield significantly higher correct categorizations compared with numerical rating scales (unipolar: $\chi^{2}(1)=32.19$; bipolar: $\chi^{2}(1)=26.82 ; p<.001$ ). The significantly higher level of categorization using semantic measures supports the validity of this method.

In the rating scales condition, the overall correctness was $74.2 \%$. Ties were categorized as incorrect, so a correct score required the rating scale score to be higher on the rating item for the targeted expression (e.g., happy) than for the two other rating scale items concerning facial expression (i.e., sad and contemptuous). This is arguably the most straightforward 
approach, especially as the validated database of pictures included prototypical facial expressions based on the Facial Action Coding System (see Langner et al., 2010). This system emphasizes the anatomy of facial expressions (e.g., Bartlett et al., 1996), where the pictures include basic emotions in which the expressions are frequently recognized across cultures (Ekman, 1992). However, a less stringent approach is to split the correctness point between the ties, so that .50 is given to answers where one of the two highest scores include the targeted expression, and .33 is given when the same rating scores have been given for all three rating scales items. Employing this approach yields an overall correct categorization of $80.7 \%$.

However, it is important to point out that affording the rating scales condition this advantage does not make it significantly better compared with the semantic measures. Training the semantic questions responses using participants as a grouping variable still produces significantly higher levels of correct categorizations $\left(\chi^{2}(1)=4.95, p=.026\right)$, whereas the comparisons with the other methods do not exhibit any significant differences. On the other hand, the semantic method could be improved by means of several other factors. For example, one could control for missing values within the semantic questions condition. That is, missing values could arguably be treated as 1/3 of a correct answer in order to become more comparable to the rating scale condition. The semantic categorization may be further improved upon by adjusting weights to each semantic similarity scale; however, this is outside the scope of this article.

To analyze inter-rater reliability, both trained and similarity scales were studied. Unipolar and bipolar scales were used for the categorization of expressions, and bipolar scales were used for the other dimensions. For the semantic trained scales of valence, intensity, clarity, genuineness and attractiveness, the semantic representations from the semantic questions were first trained to the mean score for each dimension from the rating scales condition, and then validated using leave-10\%-out cross-validation. 
Semantic measures yield significantly higher inter-rater reliability compared with rating scales with regard to both categorizing expressions and describing the related dimensions. All trained models, except for the attractiveness model grouped according to pictures, were significant in Study 2 (Pearson's $r$ ranged from .53 to .91 for the significant models $[p<.001]$; see Table S5). The inter-rater reliability statistics were measured by both Intraclass Correlation Coefficient (ICC; two-way agreement) and Krippendorff's alpha. The differences between approaches were tested by bootstrapping Krippendorff's alpha $(1,000$ iterations), followed by significance testing the results using $t$-tests between the various semantic measures and corresponding rating scale. As the bootstrapping procedure may end up computationally intensive, scores in the semantic conditions were rounded to two significant digits. Importantly, the ICC values tend to be higher using semantic measures as compared with rating scales (Table 3 ). The $t$-tests revealed that the semantic measures yield a significantly higher Krippendorff's alpha compared with the rating scales for all dimensions $(p<.001)$, except for attractiveness, where rating scales were significantly higher $(p<.001$; Table S6). Further, applying semantic predictions of ANEW valence to the semantic responses also yields a high inter-rater reliability $\left(\operatorname{ICC}_{(2,1)}=.767\right.$; Krippendorff's $\left.\alpha=.74, \mathrm{CI}=.72-76\right)$. 
Table 3

Krippendorff's Alpha $(\alpha)$ and Inter-Class Correlation Coefficient (ICC)

\begin{tabular}{|c|c|c|c|c|c|c|c|c|}
\hline \multirow{4}{*}{ Dimensions } & \multicolumn{6}{|c|}{ Three descriptive words $(\mathrm{N}: \alpha=133 ; \mathrm{ICC}=127)^{1}$} & \multicolumn{2}{|c|}{ Rating scales $(\mathbf{N}=181)$} \\
\hline & \multicolumn{4}{|c|}{ Trained semantic scales } & \multirow{2}{*}{\multicolumn{2}{|c|}{ Bipolar similarity scales }} & \multirow[b]{3}{*}{$\alpha(95 \% \mathrm{CI})$} & \multirow[b]{3}{*}{ ICC $(2,1)$} \\
\hline & \multicolumn{2}{|c|}{ Participants } & \multicolumn{2}{|c|}{ Pictures } & & & & \\
\hline & $\alpha(95 \% \mathrm{CI})$ & $\mathrm{ICC}(2,1)$ & $\alpha(95 \% \mathrm{CI})$ & $\operatorname{ICC}(2,1)$ & $\alpha(95 \% \mathrm{CI})$ & ICC $(2,1)$ & & \\
\hline Expression $^{2}$ & $.60(.52-.69)$ & NA & $.53(.44-.62)$ & NA & $\begin{array}{l}.58 ;(.49-.66)^{3} \\
.57(.48-.66)^{4}\end{array}$ & NA & $.48(.44-.52)$ & NA \\
\hline Valence & $.81(.80-.82)$ & .840 & $.81(.80-.81)$ & .806 & $.82(.81-.82)$ & .835 & $.31(.23-.38)$ & .318 \\
\hline Arousal & $.77(.75-.78)$ & .802 & $.77(.75-.78)$ & .806 & $.72(.71-.72)$ & .734 & $.13(.03-.21)$ & .137 \\
\hline Intensity & $.64(.61-.66)$ & .694 & $.62(.60-.64)$ & .667 & $.53(.51-.54)$ & .553 & $.24(.15-.32)$ & .249 \\
\hline Clarity & $.66(.64-.67)$ & .708 & $.64(.62-.65)$ & .683 & $.67(.67-.68)$ & .694 & $.24(.16-.33)$ & .255 \\
\hline Genuineness & $.77(.75-.79)$ & .817 & $.77(.75-.79)$ & .820 & $.68(.67-.69)$ & .706 & $.17(.08-.25)$ & .178 \\
\hline Attractiveness & $21(.17-.24)$ & .317 & $.01(-.09-.10)$ & .002 & $.21(.19-.22)$ & .255 & $.31(.23-.38)$ & .354 \\
\hline
\end{tabular}

Note. ${ }^{1}$ ICC is sensitive to missing values, which is why participants are removed so that these computations are based on 127 participants in the semantic question condition. ${ }^{2}$ Expressions are based on nominal data; in the semantic questions condition there are three categories; meanwhile in the rating scale condition there are seven categories including the various combinations of ties. ${ }^{3}$ Unipolar; ${ }^{4} \mathrm{Bipolar}$. 
Analyzing word responses also enables statistically based word descriptions of the constructs being studied. Figure 2 plots words that differ statistically between sets of word responses on the $\mathrm{x}$-axes and words that statistically relate to the semantic similarity scales on the y-axes. In this way, the plots describe discriminative characteristics between the various constructs in focus. And even though participants have not been primed with the psychological constructs, happy facial expressions were described as happy and joyful, sad facial expressions were described as sad and unhappy and contempt facial expressions were described as annoyed and irritated.
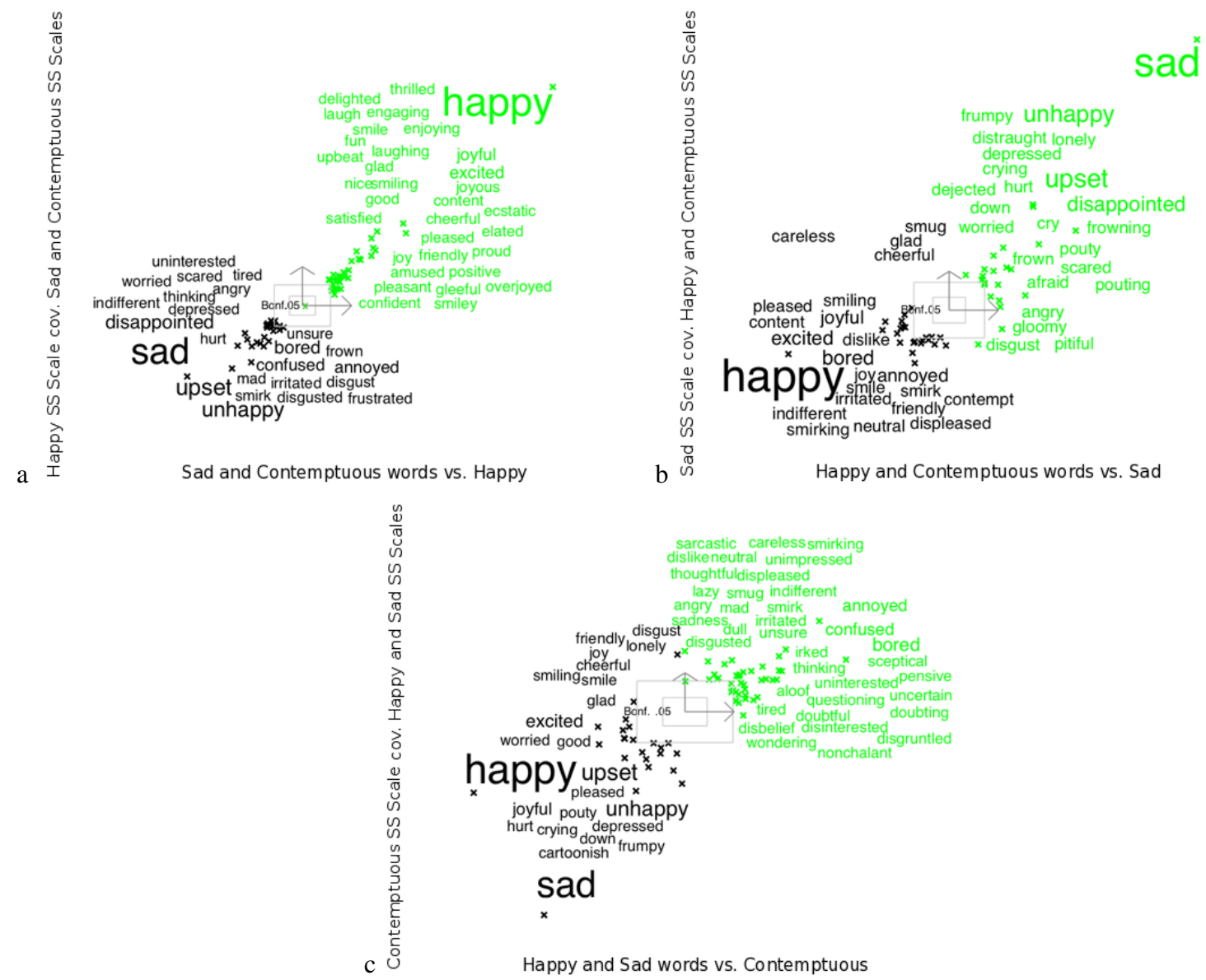

Figure $2 a-c$. Words that significantly describe one facial expression while covarying for the other two facial expressions. On the $\mathrm{x}$-axis, words are plotted according to the $\chi$-values from a Chi-square test, with Bonferroni correction for multiple comparisons (Bonf.=Bonferroni line where $\chi=4.26, .05$ indicates the uncorrected $p$-value line, where $\chi=1.96)$. On the $y$-axis, words are plotted according to point-biserial correlations $(r=.09$ at the Bonferroni line, and $\mathrm{r}=.04$ at the .05 uncorrected $p$-value line). More frequent words are plotted with a larger font size with fixed lower and upper limits. The $\mathrm{x}$-axes represent $\chi$-values associated to a) words generated to the sad 
and contemptuous pictures (black/left) versus words generated to happy pictures (green/right); in b) happy and contemptuous words (black/left) versus sad words (green/right); and in c) happy and sad words (black/left) versus contemptuous words (green/right). The y-axes show significant words related to semantic similarity (SS) scales; cov.=covariate. $\mathrm{N}=133$.

\section{Discussion}

The results from Study 2 suggest that the semantic measures encompass higher validity and inter-rater reliability compared with rating scales, except for evaluations of attractiveness; which was also supported by the results in Study 1. Even though the method used for categorizing facial expressions differed between Study 1 (using check boxes) and 2 (using rating scales), the results from the semantic conditions are to a high extent replicated between the studies. Importantly, the ability of semantic measures to correctly categorize facial expressions to a higher degree than rating scales in Study 2 demonstrates their ability to differentiate between measured constructs.

The clarification of the semantic question of attractiveness used in Study 2 revealed an improvement of inter-rater reliability from Study 1. However, the low inter-rater reliability might reflect that perceived attractiveness is subjective in nature and thus should not encompass high inter-rater reliability, as participants perceive the various models differently. That is, rating attractiveness is more subjective than categorizing prototypical facial expressions. Inter-rater reliability may potentially be further increased by additional clarification of the semantic question; for example, by making sure that all participants interpret attractiveness in a similar way. Participants may, for example, be instructed to only evaluate attractiveness rather than describing other facial characteristics they believe to be related to attractiveness. Instructions could further aim to limit the risk that participants evaluate potentially different kinds of attractiveness, such as beauty, cuteness or sexual attractiveness.

Semantic measures also have an advantage over rating scales in terms of not priming participants with concepts required for defining the numerical scales. That is, despite the fact 
that participants were not primed with the targeted expressions, happy expressions were most frequently described using the word happy and sad using the word sad. Further, previous research reports low inter-rater agreement with regard to contempt and has argued that this is the result of issues with the label of the expression rating scale rather than the expression itself (Langner et al., 2010). In contrast, the semantic questions approach lets participants generate a description of the expression, including annoyed and irritated. This means that whereas rating scales only provide a number in relation to the question/item, semantic measures enhance the understanding of what was measured by providing a description of it. In sum, the results demonstrate that the semantic measures may be used for measuring, differentiating and describing psychological dimensions of facial expressions.

\section{Reports regarding Subjective States}

Next, we develop semantic measures for two psychological constructs pertaining to well-being: harmony in life and satisfaction with life, as well as two psychological constructs relating to mental health problems: depression and worry. These constructs are theoretically different, but are frequently found to be highly correlated when measured by rating scales. In these studies, the answers have no relation to external stimuli identical to all participants, which was the case in the previous facial expression studies. Instead, we analyze how the self-reported subjective responses relate to each other across psychological constructs and response formats (i.e., semantic questions versus rating scales).

Satisfaction with life, here measured with the numerical rating scale the Satisfaction with Life Scale (SWLS; Diener et al., 1985), focuses on evaluations based on comparing actual with expected and desired life circumstances. Harmony in life is here measured using the numerical rating scale the Harmony in Life Scale (HILS; Kjell et al., 2015). Li (2008) points out that: "Harmony is by its very nature relational. It is through mutual support and mutual dependence that things flourish" (p. 427). Hence, the HILS focuses on psychological balance 
and interconnectedness with those aspects considered important for the respondent. Kjell et al. (2015) found that harmony in life and satisfaction with life differ significantly in terms of how participants perceive their pursuit of each construct. The pursuit of harmony in life is significantly related to words linked to interdependence and being at peace such as peace, balance, calm, unity and love, whereas the pursuit of satisfaction with life is significantly related to words linked to independence and achievements, such as money, work, career, achievement and fulfillment. Hence, these (and associated) words are proposed to make up the positive endpoints representing a high degree of harmony in life versus satisfaction with life, respectively. Further, the words generated in relation to each construct significantly differed with a medium effect size (Cohen's $\mathrm{d}=.72$ ) in a semantic $t$-test (Kjell et al., 2015). This clear semantic difference between the constructs suggests that semantic measures might provide a clear differentiation between the two constructs.

Depression and worry/anxiety share a common mood factor of negative affectivity, whereas low positive affectivity is typical for depression alone (Axelson \& Birmaher, 2001; Brown, Chorpita \& Barlow, 1998; Watson, Clark \& Carey, 1988). Clinical depression is characterized by symptoms such as a lack of interest or pleasure in doing things, fatigue, feelings of hopelessness and sadness (American Psychiatric Association, 2013). Depression is often measured by the nine-item Patient Health Questionnaire (PHQ-9), which targets the DSM-IV diagnostic criteria (Kroenke, Spitzer \& Williams, 2001). We anticipate that the semantic responses of participants in relation to depression correspond to these criteria (including words such as sad, tired, disinterested, etc.). Excessive and uncontrollable worry, on the other hand, is recognized by symptoms such as restlessness, being on edge and irritability (American Psychiatric Association, 2013). Worry is often assessed with the sevenitem Generalized Anxiety Disorder scale (GAD-7; Spitzer, Kroenke, Williams \& Löwe, 2006), 
which is linked to these symptoms. Thus, semantic responses to worry are anticipated to relate to these symptoms (including words such as tense, nervous, anxious, etc.).

Numerical rating scales targeting depression and worry/anxiety tend to correlate strongly with each other (Muntingh et al., 2011; Spitzer et al., 2006). This may be seen as a measurement problem associated with numerical rating scales, as correctly identifying and differentiating between the two constructs becomes difficult. Some argue that this significant overlap is due to a frequent co-occurrence between these conditions (Kessler, Chiu, Demler, Merikangas \& Walters, 2005), whereas others argue that problems differentiating these have considerable implications in terms of treatment (Brown, 2007; Wittchen et al., 2002). However, considering the conceptual and criteria-based differences between these two constructs, semantic measures are proposed to be able to differentiate between these constructs more clearly than rating scales.

\section{The Overall Rationale of the Studies concerning Subjective States}

Studies 3-9 focused on reports regarding subjective states, where participants answered semantic questions by generating descriptive words or texts. Participants then answered numerical rating scales corresponding to the studied constructs. The semantic questions were presented first so that the items in the rating scales would not influence the generation of words and texts by participants. Since we are developing a new approach for measuring subjective states, we have carried out seven studies, which in a controlled and iterative manner enabled us to examine potential strengths and weaknesses associated with the method, as well as the replicability of the main findings (which is important considering the replicability concerns within psychological research; e.g., Open Science Collaboration, 2015).

The aim of Study 3 was to pilot the semantic questions of satisfaction with life and harmony in life and study their relationships with the corresponding numerical rating scales. The aim of Study 4 was to examine the semantic questions in a larger sample than the one used 
in Study 3. The aim of Study 5 involved examining how the semantic measures of harmony and satisfaction relate to rating scales of depression, anxiety and stress. Studies 3-5 included both descriptive words and text responses. The aim of Study 6 was to test shorter instructions for the semantic questions. The aim of Study 7 was to examine the effects of requiring fewer word responses, where participants only had to answer the semantic questions using one, three or five words rather than ten. The aim of Study 8 was to develop semantic questions for depression and worry. The aims of Study 9 involved examining the test-retest reliability of the semantic measures for harmony and satisfaction, and at time 2 (T2) examine the interrelationship between the semantic measures for all four constructs.

\section{Methods}

\section{Participants}

Participants were recruited using Mechanical Turk. The samples for the studies concerning subjective states are described in Table 4.

Table 4

Information about Participants within each Study for Subjective States

\begin{tabular}{|c|c|c|c|c|c|c|}
\hline $\begin{array}{l}\text { Study: } \\
\text { Condition }\end{array}$ & $\begin{array}{l}\mathbf{N} \\
\text { (excluded } \\
\text { due to } \\
\text { control } \\
\text { questions) }\end{array}$ & $\begin{array}{l}\text { Age } \\
\text { Min- } \\
\text { Max; } \\
\text { Mean } \\
\text { (SD) } \\
\text { years }\end{array}$ & Gender & Nationality & $\begin{array}{l}\text { Mean time } \\
\text { (SD) in } \\
\text { minutes } \\
\text { and } \\
\text { seconds }\end{array}$ & $\begin{array}{c}\text { Payment } \\
\text { in US\$ }\end{array}$ \\
\hline Study 3. & $92(13)$ & $\begin{array}{l}\text { 18-64; } \\
33.36 \\
(10.93)\end{array}$ & $\begin{array}{l}\mathrm{F}=51.1 \% \\
\mathrm{M}=48.9 \%\end{array}$ & $\begin{array}{l}\mathrm{US}=73.9 \% \\
\mathrm{IN}=23.9 \% \\
\mathrm{O}=2.2 \%\end{array}$ & $\begin{array}{l}14.18 \\
(8.23)\end{array}$ & 0.30 \\
\hline Study 4. & $303(24)$ & $\begin{array}{l}18-74 ; \\
34.87 \\
(12.17)\end{array}$ & $\begin{array}{l}\mathrm{F}=55.4 \% \\
\mathrm{M}=44.6 \%\end{array}$ & $\begin{array}{l}\mathrm{US}=95 \% \\
\mathrm{IN}=2.6 \% \\
\mathrm{O}=2.3 \%\end{array}$ & $\begin{array}{l}20.06 \\
(10.20)\end{array}$ & 0.50 \\
\hline Study 5. & $296(19)$ & $\begin{array}{l}18-74 ; \\
36.40 \\
(13.45)\end{array}$ & $\begin{array}{l}\mathrm{F}=60.1 \% \\
\mathrm{M}=39.9 \%\end{array}$ & $\begin{array}{l}\text { US }=86.1 \% \\
\mathrm{IN}=10.5 \% \\
\mathrm{O}=3.4 \%\end{array}$ & $\begin{array}{l}17.08 \\
(21.10)\end{array}$ & 0.30 \\
\hline Study 6. & $193(9)$ & $\begin{array}{l}18-64 ; \\
35.88 \\
(10.97)\end{array}$ & $\begin{array}{l}\mathrm{F}=51.8 \% \\
\mathrm{M}=48.2 \%\end{array}$ & $\begin{array}{l}\text { US }=78.8 \% \\
\mathrm{IN}=18.1 \% \\
\mathrm{O}=3.1 \%\end{array}$ & $\begin{array}{l}10.27 \\
(7.07)\end{array}$ & 0.80 \\
\hline Study 7: 1-word & $361(20)$ & $\begin{array}{l}18-72 ; \\
30.80 \\
(10.02)\end{array}$ & $\begin{array}{l}\mathrm{F}=43.8 \% \\
\mathrm{M}=56.0 \% \\
\mathrm{O}=0.3 \%\end{array}$ & $\begin{array}{l}\mathrm{US}=91.4 \% \\
\mathrm{IN}=6.4 \% \\
\mathrm{O}=2.2 \%\end{array}$ & $3.07(2.50)$ & 0.20 \\
\hline
\end{tabular}




\begin{tabular}{|c|c|c|c|c|c|c|}
\hline Study 7: 3-words & $350(18)$ & $\begin{array}{l}18-65 \\
31.61 \\
(9.65)\end{array}$ & $\begin{array}{l}\mathrm{F}=48.6 \% \\
\mathrm{M}=50.9 \% \\
\mathrm{O}=0.6 \%\end{array}$ & $\begin{array}{l}\text { US }=95.4 \% \\
\mathrm{IN}=2.6 \% \\
\mathrm{O}=2.0 \%\end{array}$ & $3.35(2.23)$ & 0.20 \\
\hline Study 7: 5-words & $257(19)$ & $\begin{array}{l}18-63 \\
30.53 \\
(9.24)\end{array}$ & $\begin{array}{l}\mathrm{F}=44.4 \% \\
\mathrm{M}=55.6 \%\end{array}$ & $\begin{array}{l}\text { US }=94.2 \% \\
\mathrm{IN}=3.5 \% \\
\mathrm{O}=2.3 \%\end{array}$ & $4.34(4.47)$ & 0.20 \\
\hline Study 8. & 399 (36) & $\begin{array}{l}18-69 ; \\
34.45 \\
(11.45)\end{array}$ & $\begin{array}{l}\mathrm{F}=51.0 \% \\
\mathrm{M}=48.7 \% \\
\mathrm{O}=0.3 \%\end{array}$ & $\begin{array}{l}\text { US }=86.2 \% \\
\mathrm{IN}=10.6 \% \\
\mathrm{O}=3.3 \%\end{array}$ & $9.44(5.42)$ & 0.50 \\
\hline Study 9: T1 & $854(42)$ & $\begin{array}{l}18-64 ; \\
32.76 \\
(10.09)\end{array}$ & $\begin{array}{l}\mathrm{F}=50.9 \% \\
\mathrm{M}=49.0 \%\end{array}$ & $\begin{array}{l}\text { US }=93.5 \% \\
\mathrm{IN}=4.1 \% \\
\mathrm{O}=2.1 \%\end{array}$ & $5.53(3.52)$ & 0.50 \\
\hline Study 9: T2 & $477(42)$ & $\begin{array}{l}18-63 ; \\
34.11 \\
(10.47)\end{array}$ & $\begin{array}{l}\mathrm{F}=54.5 \% \\
\mathrm{M}=45.5 \%\end{array}$ & $\begin{array}{l}\mathrm{US}=93.9 \% \\
\mathrm{IN}=4.4 \% \% \\
\mathrm{O}=1.7 \%\end{array}$ & $\begin{array}{l}16.32 \\
(9.32)\end{array}$ & 1.00 \\
\hline \multicolumn{7}{|l|}{ Word-Norms ${ }^{1}$} \\
\hline Harmony & 120 & $\begin{array}{l}18-51 ; \\
29.43 \\
(7.89)\end{array}$ & $\begin{array}{l}\mathrm{F}=40.8 \% \\
\mathrm{M}=59.2 \%\end{array}$ & $\begin{array}{l}\text { US }=95.8 \% \\
\mathrm{IN}=1.7 \% \\
\mathrm{O}=2.5 \%\end{array}$ & $2.46(2.16)$ & 0.10 \\
\hline Disharmony & 96 & $\begin{array}{l}18-59 \\
29.75 \\
(8.49)\end{array}$ & $\begin{array}{l}\mathrm{F}=43.8 \% \\
\mathrm{M}=56.3 \%\end{array}$ & $\begin{array}{l}\text { US }=93.8 \% \\
\mathrm{IN}=5.2 \% \\
\mathrm{O}=1.0 \%\end{array}$ & $2.36(1.28)$ & 0.10 \\
\hline Satisfaction & 93 & $\begin{array}{l}19-60 \\
29.87 \\
(9.12)\end{array}$ & $\begin{array}{l}\mathrm{F}=31.2 \% \\
\mathrm{M}=68.8 \%\end{array}$ & $\begin{array}{l}\mathrm{US}=98.9 \% \\
\mathrm{O}=1.1 \%\end{array}$ & $2.15(1.07)$ & 0.10 \\
\hline Dissatisfaction & 84 & $\begin{array}{l}18-74 ; \\
33.14 \\
(13.35)\end{array}$ & $\begin{array}{l}\mathrm{F}=44 \% \\
\mathrm{M}=56 \%\end{array}$ & $\begin{array}{l}\text { US }=91.7 \% \\
\mathrm{IN}=4.8 \% \\
\mathrm{O}=3.6 \%\end{array}$ & $2.44(1.41)$ & 0.10 \\
\hline Worried & 104 & $\begin{array}{l}18-65 \\
28.73 \\
(8.80)\end{array}$ & $\begin{array}{l}F=52.9 \% \\
M=46.2 \% \\
O=1.0 \%\end{array}$ & $\begin{array}{l}\text { US }=93.3 \% \\
\mathrm{IN}=4.8 \% \\
\mathrm{O}=1.9 \%\end{array}$ & $2.14(1.46)$ & 0.10 \\
\hline Depressed & 110 & $\begin{array}{l}18-65 \\
30.57 \\
(9.64)\end{array}$ & $\begin{array}{l}\mathrm{F}=45.5 \% \\
\mathrm{M}=53.6 \% \\
\mathrm{O}=0.9 \%\end{array}$ & $\begin{array}{l}\text { US }=89.1 \% \\
\mathrm{IN}=7.3 \% \\
\mathrm{O}=3.6 \%\end{array}$ & $1.59(1.52)$ & 0.10 \\
\hline
\end{tabular}

Note. $\mathrm{F}=$ Female; $\mathrm{M}=$ Male; $\mathrm{O}=\mathrm{Other}$; US= United States of America; IN=India. ${ }^{1}$ In Word-Norms studies there were no control questions.

\section{Measures}

Semantic questions concerning subjective states were developed for both using descriptive words and text responses. Pilot studies from our lab have shown that a high validity of the semantic measures requires a semantic question with instructions to be posed in a clear manner. Hence, the following guidelines were applied: Participants should in detail describe their state or perception of the to-be-answered question, for example their own life satisfaction, rather 
than describing their view of life satisfaction as a general concept. To get the best effect, the question and its accompanying instructions should stress the strength and frequency of words related to the construct (or lack of it). To receive a consistent response mode among participants, the instructions should also inform the participant to write one word in each box (except for the text responses) to discourage freestanding negations (e.g., "not happy" rather than "unhappy" or "sad"). The questions, which in combination with instructions were used for prompting open-ended responses within the studies, include: "Overall in your life, are you in harmony or not?"; "Overall in your life, are you satisfied or not?"; "Over the last 2 weeks, have you been worried or not?" and "Over the last 2 weeks, have you been depressed or not?" The questions are posed with different time frames (i.e., overall in your life versus over the last 2 weeks) to reflect the timeframes prompted in the instructions/items for each respective numerical rating scale. The instructions for the semantic questions concerning harmony are presented below (these were then adapted to satisfaction with life, depression and worry).

Semantic question instructions for descriptive words responses: "Please answer the question by writing 10 descriptive words below that indicate whether you are in harmony or not. Try to weigh the strength and the number of words that describe if you are in harmony or not so that they reflect your overall personal state of harmony. For example, if you are in harmony then write more and stronger words describing this, and if you are not in harmony then write more and stronger words describing that. Write descriptive words relating to those aspects that are most important and meaningful to you. Write only one descriptive word in each box."

Short semantic question instructions for descriptive words responses: "Please answer the question by writing 10 words that describe whether you are in harmony or not. Write only one descriptive word in each box." 
Semantic question instructions for text responses: "Please answer the question by writing at least a paragraph below that indicates whether you are in harmony or not. Try to weigh the strength and the number of aspects that describe if you are in harmony or not so that they reflect your overall personal state of harmony. For example, if you are in harmony then write more about aspects describing this, and if you are not in harmony then write more about aspects describing that. Write about those aspects that are most important and meaningful to you."

Word norm items for subjective states included the following instructions: "Please write 10 words that best describe your view of harmony in life. Write descriptive words relating to those aspects that are most important and meaningful to you. Write only one descriptive word in each box." The instructions were adapted to also cover "disharmony in life," "satisfaction with life," "dissatisfaction with life," "being worried" and "being depressed." (As there are no antonyms for worried and depressed, norms for these constructs were not created for the current study, although one could potentially create norms for "not worried" and "not depressed".) The targeted words were also added (i.e., $\mathrm{f}(\max )+1)$, as in the facial expression studies.

The Harmony in Life Scale (Kjell et al., 2015) was used in study 3-7 and 9. The scale includes five items (e.g., "I am in harmony"), which are answered on a scale ranging from 1=“Strongly Disagree" to 7="Strongly Agree." For the different studies, McDonald's $\omega$ (Dunn, Baguley \& Brunsden, 2014) ranged from .91-.95 and Cronbach's $\alpha$ ranged from .89-.95.

The Satisfaction with Life Scale (Diener et al., 1985) was used in study 3-7 and 9. It comprises five items (e.g., "I am satisfied with life") answered on the same scale as the HILS. In the various studies, McDonald's $\omega$ and Cronbach's $\alpha$ ranged from .90 to .94 .

The Depression, Anxiety and Stress Scale, the short version (DASS; Sinclair et al., 2012) was used in Study 5. It includes seven items for each of the three constructs: depression (e.g., "I felt downhearted and blue"), anxiety (e.g., "I felt I was close to panic") and stress (e.g., 
"I found it hard to wind down"). Participants were required to answer using a 4-point scale assessing severity/frequency of the constructs. Both McDonald's $\omega$ and Cronbach's $\alpha$ were .93 for depression and .90 for anxiety and stress.

The Generalized Anxiety Disorder Scale-7 (Spitzer et al., 2006) was used for measuring worry in Study 8 and 9 T2. It includes seven items answered on a scale ranging from $0=$ "Not at all" to $3=$ "Nearly every day." It assesses how frequently the respondent has been bothered by various problems over the last two weeks (e.g., "Worrying too much about different things"). In both studies, McDonald's $\omega$ and Cronbach's $\alpha$ were .94 .

The Patient Health Questionnaire-9 (Kroenke \& Spitzer, 2002) was used for assessing depression in Study 8 and 9 at T2. Its structure and response format is similar to that of GAD7, comprising nine items (e.g., "Feeling down, depressed or hopeless"). In both studies, McDonald's $\omega$ and Cronbach's $\alpha$ were .93 .

The Marlowe-Crowne Social Desirability Scale (Crowne \& Marlowe, 1960) the short version Form A (MC-SDS-FA; Reynolds, 1982) was used in Study 9 at T2. It encompasses twelve items (e.g., "No matter who I'm talking to, I'm always a good listener"), which require responses indicating whether the statements are personally true or false. Both McDonald's $\omega$ and Cronbach's $\alpha$ were .73.

Control Questions were used in accordance to previous research (Kjell et al., 2015). These required respondents in study 3-9 to answer a specific alternative (e.g., "Answer 'disagree' on this question") in various places throughout the surveys. Participants who did not answer all control items correctly were excluded from the analyses, as this type of approach has been demonstrated to yield high statistical power and improve reliability (Oppenheimer, Meyvis \& Davidenko, 2009). 


\section{Procedures}

In Study 3-9 regarding subjective states, the semantic questions were presented first followed by the rating scales (see Table 5 for an overview of the type of semantic questions and rating scales included in these studies). Study 3-7 started with the semantic questions concerning harmony in life and satisfaction with life in a random order. Only Study 3-5 included both descriptive words and descriptive texts as response formats. Participants were randomly presented with either the word- or text-based items first (harmony and satisfaction were in random order). Study 6-9 only included descriptive word-based (not text) response formats. Study 6 included the short instruction version of the descriptive word item. In Study 7, participants were asked to answer using either one, three or five words.

In study 3-7 and 9, the open-ended items were followed by the HILS and the SWLS. The DASS was presented last in Study 5. Study 8 encompassed the same general structure as the one used in previous studies, but instead of asking about harmony and satisfaction, it included semantic questions concerning worry and depression, followed by the respective rating scales: the GAD-7 and the PHQ-9.

Study 9 involved a test-retest procedure. This meant that at time 1 (T1), participants filled out the semantic questions for harmony and satisfaction followed by the HILS and the SWLS. At T2, they were asked via the message service of Mechanical Turk to partake in a follow-up study. At T2 (30.79, SD=2.01, days after T1), they first filled out the questions from T1 followed by the questions from Study 8. Finally, they filled out the MC-SDS-FA.

In the development of the word norms for subjective states, participants were randomly assigned to answer one of the word norm questions and then answer the demographic battery of questions. The studies received ethical approval from the ethical committee in Lund, Sweden. 
Table 5.

Types of Semantic Questions and Numerical Rating Scales Included in each of the Studies

\begin{tabular}{|c|c|c|}
\hline Study & Semantic Questions & Numerical Rating Scales \\
\hline 3 & H \& S; words and text responses & HILS, SWLS \\
\hline $4^{\mathrm{a}}$ & $\mathrm{H} \& \mathrm{~S}$; words and text responses & HILS, SWLS \\
\hline 5 & $\mathrm{H} \& \mathrm{~S}$; words and text responses & HILS, SWLS, DASS \\
\hline 6 & H \& S; words responses; short instructions & HILS, SWLS \\
\hline 7 & H \& S; $\mathbf{1}, \mathbf{3}$ or 5 words responses & HILS, SWLS \\
\hline 8 & D \& W; words responses & GAD-7, PHQ-9 \\
\hline 9: T1 & H \& S; words responses & HILS, SWLS \\
\hline 9: $\mathbf{T 2}$ & $\mathrm{H}, \mathrm{S}, \mathrm{D} \& \mathrm{~W}$; words responses & HILS, SWLS, GAD-7, PHQ-9, MC-SDS-FA \\
\hline \multicolumn{3}{|c|}{$\begin{array}{l}\text { Note. The semantic questions included the long instructions and required } 10 \text { descriptive words if nothing else is } \\
\text { specified. Bold font highlights important (new) aspects of the study; }{ }^{a}=\text { Study } 4 \text { includes more participants than } \\
\text { Study } 3 . \mathrm{H}=\text { Harmony in life; } \mathrm{S}=\text { Satisfaction with life; } \mathrm{D}=\text { Depression; } \mathrm{W}=\text { Worry; HILS = Harmony in life } \\
\text { scale; SWLS = Satisfaction with life scale; DASS = Depression, Anxiety and Stress Scales the short version; } \\
\text { GAD-7 = Generalized anxiety disorder scale-7; PHQ-9 = Patient Health Questionnaire-9; MC-SDS-FA = The } \\
\text { Marlowe-Crowne Social Desirability Scale, the short version Form A }\end{array}$} \\
\hline
\end{tabular}

\section{Results}

Descriptive data regarding the number of words generated by participants for the semantic questions (Table S7) and the results for the descriptive text responses are presented in the SM. Some semantic measures and rating scales were not normally distributed; in particular GAD-7 and PHQ-9 showed a positive skew (which makes sense, since these scales were originally developed for a clinical population, but have later been validated in the general population [see Löwe et al., 2008 for GAD-7 and Martin, Rief, Klaiberg \& Braehler, 2006 for PHQ-9]). Consequently, it could be argued that rank-ordered statistical analyses, such as Spearman's rho, should be used in these instances. Throughout this article, however, Pearson's $r$ is presented as being consistent in the studies on subjective reports and thus increase comparability across studies. Further, Pearson's $r$ does not involve transforming the data into ranks and thus losing information. Importantly though, computing Spearman's rho tends to yield similar results and overall conclusions as compared with Pearson's r (e.g., with Spearman's rho in Study 9 at T2, there tends to be slightly smaller correlation coefficients for the well-being measures and larger coefficients for the ill-being measures). 


\section{Predicting Rating Scale Scores from Semantic Word Responses}

Using semantic-numeric correlations, the predictive validity of semantic word responses was examined in relation to the rating scales. Table 6 shows that the semantic responses may be trained to predict targeted numerical rating scales (i.e., the semantic responses for harmony predict the targeted rating scale the HILS rather than, for example, the SWLS) with consistently high correlations. In Study $9(N=477)$, the predictions yielded strong correlations to targeted rating scale scores $(\mathrm{r}=.58-.72, p<.001$; Table 6$)$. Further, results show that the semantic responses of harmony and satisfaction may also be used for predicting rating scores of depression, anxiety and stress, although with lower correlations compared with the targeted numerical rating scale (Table S9). It is here worth noting that these lower correlations show that the trained scales tend to discriminate between psychological constructs insofar that the semantic responses predict the rating scales of the targeted construct better than other constructs.

Even though the SWLS and the HILS ( $\mathrm{r}=.80$ to $\mathrm{r}=.88$ ) as well as the GAD-7 and the PHQ-9 ( $r=.86$ to $r=.87)$ correlate strongly throughout the studies, the semantic responses tend to differentiate among these constructs. In Study 8 and 9 at T2, semantic responses regarding depression are trained to best predict the rating scale of depression, whilst semantic responses of worry are trained to best predict the rating scale of worry. Similarly, in Study 3-9, the semantic responses regarding harmony in life are trained to best predict the rating scale of harmony. However, note that this prediction specificity (i.e., that word responses predict the targeted rating scale score better than other rating scale scores) is not consistent with regard to satisfaction. The semantic word responses regarding satisfaction with life are trained to predict rating scales of satisfaction and harmony equally well in Study 5. In Study 6 and 7, where one and three words were required as a response, as well as Study 9 at T2, the satisfaction responses actually predict the rating scale of harmony better than the rating scale of satisfaction. Hence, 
semantic responses concerning harmony consistently predict the rating scale of harmony better than the rating scale of satisfaction, whereas semantic content of satisfaction appears to predict both rating scales equally well. As the purpose of training is to find the best mathematical fit rather than providing a theoretical explanation, the underlying theoretical reason behind this requires more research. In short, the overall tendency for prediction specificity (e.g., where harmony responses predict the HILS better than the SLWS) throughout the studies and across the constructs (except for satisfaction) support the validity of the semantic responses and their semantic representations.

The trained predictions are also robust (Table 7-9). Examining the correlations as a function of participants shows that a sample size of approximately 64 participants is required to reach significant results, although the correlations increase with sample size (Table 7). Examining the function of generated words reveals that the first word accounts for the largest part of the predictive power (Table 8) and that the ninth and tenth words do not always increase the correlation (Table 9). To sum up, that semantic responses reliably predict rating scales supports the validity of the semantic measures. 
Table 6

Semantic Responses Trained to Rating Scales Scores Demonstrate Validity, Reliability and the High Influence of Valence

\begin{tabular}{|c|c|c|c|c|c|c|c|c|c|c|c|c|c|c|}
\hline \multicolumn{2}{|c|}{ Study: condition } & $\begin{array}{l}\mathrm{S3} \\
\mathrm{N}=91\end{array}$ & $\begin{array}{l}\mathrm{S} 4 \\
\mathrm{~N}=301\end{array}$ & $\begin{array}{l}\text { S5 } \\
N=294\end{array}$ & $\begin{array}{l}\text { S6 } \\
N=190\end{array}$ & $\begin{array}{l}\text { S7: 1-w } \\
N=355\end{array}$ & $\begin{array}{l}\text { S7: 3-w } \\
N=349\end{array}$ & $\begin{array}{l}\text { S7: 5-w } \\
N=256\end{array}$ & $\begin{array}{l}\text { S9: } T 1 \\
N=852\end{array}$ & $\begin{array}{l}\text { S9: T2 } \\
\text { N=477 }\end{array}$ & & & $\begin{array}{l}\text { S8 } \\
N=399\end{array}$ & $\begin{array}{l}\text { S9: } T 2 \\
N=477\end{array}$ \\
\hline \multicolumn{2}{|c|}{ Correlation items } & \multicolumn{8}{|c|}{ Pearson's $\mathbf{r}$} & \multicolumn{3}{|c|}{ Correlation items } & \multicolumn{2}{|c|}{ Pearson's $r$} \\
\hline \multirow[t]{2}{*}{ Hw } & HILS & $.60 * * *$ & $.66 * * *$ & $.71 * * *$ & $.60 * * *$ & $.56 * * *$ & $.57 * * *$ & $.58 * * *$ & $.70 * * *$ & $.72 * * *$ & Ww & GAD-7 & $.57 * * *$ & $.58 * * *$ \\
\hline & SWLS & $.34 * * *$ & $.49 * * *$ & $.61 * * *$ & $.50 * * *$ & $.33 * * *$ & $.36 * * *$ & $.47 * * *$ & $.62 * * *$ & $.48 * * *$ & & PHQ-9 & $.47 * * *$ & $.43 * * *$ \\
\hline \multirow{2}{*}{$\begin{array}{l}\text { Hw: Valence } \\
\text { as covariate }\end{array}$} & HILS & ns & ns & $.23 * * *$ & ns & $.36 * * *$ & ns & $.19 * *$ & $.30 * * *$ & $.17 * * *$ & Ww: Valence & GAD-7 & $.14 * *$ & $.12 * *$ \\
\hline & SWLS & ns & ns & ns & ns & $.16^{* *}$ & ns & $.11 *$ & $.22 * * *$ & ns & as covariate & PHQ-9 & ns & ns \\
\hline \multirow[t]{2}{*}{ Sw } & SWLS & $.50 * * *$ & $.66^{* * *}$ & $.62 * * *$ & $.58 * * *$ & $.47 * * *$ & $.56^{* * *}$ & $.60 * * *$ & $.70 * * *$ & $.63 * * *$ & Dw & PHQ-9 & $.63 * * *$ & $.59 * * *$ \\
\hline & HILS & ns & $.64 * * *$ & $.62 * * *$ & $.68 * * *$ & $.50 * * *$ & $.58 * * *$ & $.52 * * *$ & $.69 * * *$ & $.68 * * *$ & & GAD-7 & $.56 * * *$ & $.51 * * *$ \\
\hline \multirow{2}{*}{$\begin{array}{l}\text { Sw: Valence } \\
\text { as covariate }\end{array}$} & SWLS & $.25^{* *}$ & $.12 *$ & ns & ns & $.27 * * *$ & $.18 * * *$ & ns & $.19 * * *$ & $.09 *$ & Dw: Valence & PHQ-9 & $.20 * * *$ & ns \\
\hline & HILS & ns & ns & ns & $.25 * * *$ & $.34 * * *$ & ns & ns & $.10 * *$ & $.21 * * *$ & as covariate & GAD-7 & ns & ns \\
\hline HILS & SWLS & $.80 * * *$ & $.84 * * *$ & $.88 * * *$ & $.84 * * *$ & $.83^{* * *}$ & $.83 * * *$ & $.84 * * *$ & $.84 * * *$ & $.83 * * *$ & GAD-7 & PHQ-9 & $.86 * * *$ & $.87 * * *$ \\
\hline
\end{tabular}

Note. Pearson's $\mathrm{r}$ between predicted and actual values. ns $=$ not significant, $*=\mathrm{p}<.05 ; * *=\mathrm{p}<.01 ; * * *=\mathrm{p}<.001$. Hw=harmony words; Sw $=$ Satisfaction words; Ww $=$ worry; Dw=Depression words; HILS=Harmony in Life Scales; SWLS=Satisfaction with Life Scales; GAD-7=Generalized Anxiety Disorder Scale-7; PHQ-9=Patient Health Questionnaire-9. S=Study; 1-w, 3-w and 5-w = number of words required as response to the semantic question (i.e., one, three and five respectively). 
Table 7

Correlations between Predicted and Actual Rating Scale Scores as a Function of Number of Participants in Study 9 at T2

\begin{tabular}{|c|c|c|c|c|}
\hline \multirow[t]{2}{*}{ N-ps } & \multicolumn{4}{|c|}{$\begin{array}{c}\text { Function of N participants } \\
\text { Pearson's } r\end{array}$} \\
\hline & $\begin{array}{l}\text { Hw: } \\
\text { HILS }\end{array}$ & $\begin{array}{l}\text { Sw: } \\
\text { SWLS }\end{array}$ & $\begin{array}{l}\text { Ww: } \\
\text { GAD-7 }\end{array}$ & $\begin{array}{l}\text { Dw: PHQ- } \\
9\end{array}$ \\
\hline 8 & ns & ns & ns & ns \\
\hline 16 & ns & ns & ns & ns \\
\hline 32 & $.40 * *$ & $.20 *$ & ns & $.30 *$ \\
\hline 64 & .48 & .43 & $.25^{* *}$ & .21 \\
\hline 128 & .63 & .46 & .47 & .39 \\
\hline 256 & .70 & .62 & .51 & .49 \\
\hline 477 & .72 & .63 & .58 & .59 \\
\hline \multicolumn{5}{|c|}{$\begin{array}{l}\text { Note. All correlations were significant at } \mathrm{p}<.001 \\
\text { unless otherwise specified, } *=\mathrm{p}<.05, * *=\mathrm{p}<.01 \text {, } \\
\text { ns=not significant, } \quad(\mathrm{N}=477) . \quad \text { ws=words; } \\
\text { ps=participants; Hw=Harmony } \quad \text { words; } \\
\text { Sw=Satisfaction words; Ww=Worry words; } \\
\text { Dw=Depression words; HILS=Harmony in Life } \\
\text { Scales; SWLS=Satisfaction with Life Scales; GAD- } \\
\text { 7=Generalized Anxiety Disorder Scale-7; PHQ- } \\
\text { 9=Patient Health Questionnaire-9. }\end{array}$} \\
\hline
\end{tabular}

Table 8

Correlations Between Predicted and Actual Rating Scale Scores as a Function of Serial Position of the Words in Study 9 at T2

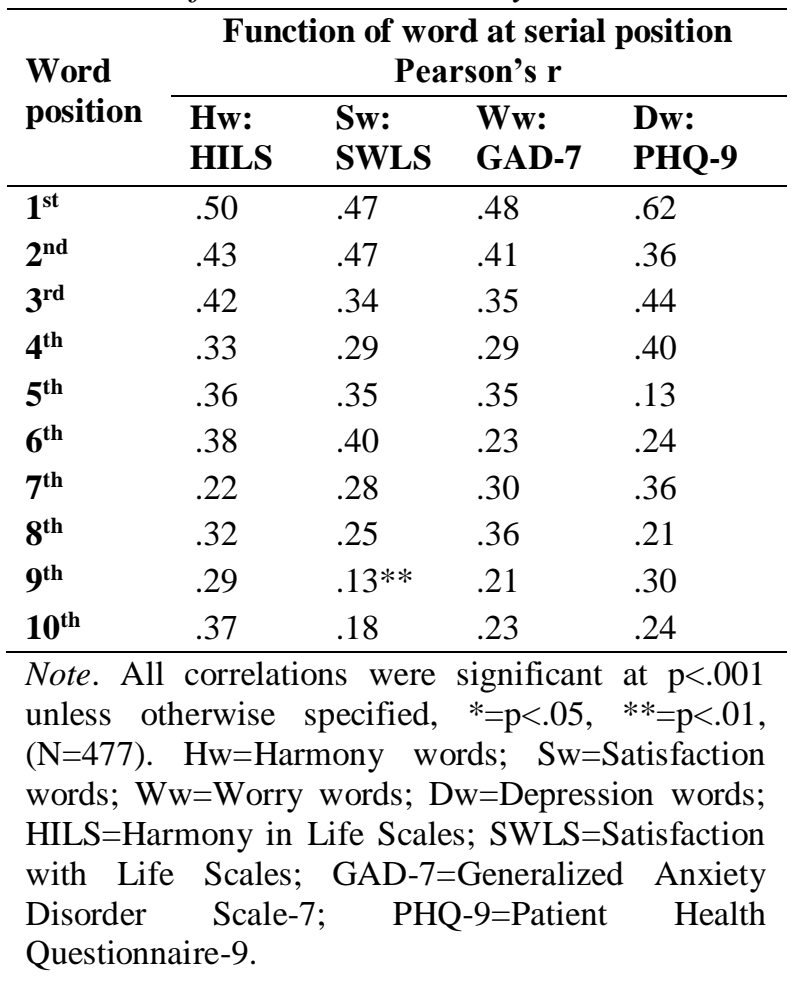


Table 9

Correlations between Predicted and

Actual Rating Scale Scores as a Function

of the Number of Words in Study 9 at T2

\begin{tabular}{lllll}
\hline \multirow{2}{*}{$\begin{array}{l}\text { N- } \\
\text { words }\end{array}$} & \multicolumn{4}{c}{$\begin{array}{c}\text { Function of N-first-words } \\
\text { Pearson's r }\end{array}$} \\
\cline { 2 - 5 } & Hw: & Sw: & Ww: & Dw: \\
& HILS & SWLS & GAD-7 & PHQ-9 \\
\hline $\mathbf{1}$ & .50 & .47 & .48 & .62 \\
$\mathbf{2}$ & .62 & .56 & .48 & .50 \\
$\mathbf{3}$ & .66 & .58 & .45 & .57 \\
$\mathbf{4}$ & .62 & .58 & .51 & .60 \\
$\mathbf{5}$ & .67 & .62 & .54 & .59 \\
$\mathbf{6}$ & .66 & .62 & .57 & .61 \\
$\mathbf{7}$ & .66 & .64 & .57 & .63 \\
$\mathbf{8}$ & .67 & .64 & .59 & .62 \\
$\mathbf{9}$ & .69 & .63 & .59 & .61 \\
$\mathbf{1 0}$ & .72 & .63 & .58 & .59 \\
\hline Note. All correlations were significant at p<.001 \\
unless otherwise specified, *=p<.05, **=p<.01, \\
(N=477). Hw=Harmony words; Sw=Satisfaction \\
words; Ww=Worry words; Dw=Depression \\
words; HILS=Harmony in Life Scales; \\
SWLS=Satisfaction with Life Scales; GAD- \\
7=Generalized Anxiety Disorder Scale-7; PHQ- \\
9=Patient Health Questionnaire-9.
\end{tabular}

\section{Semantic Similarity Scales Independently Measure Constructs}

Semantic similarity scales may be used for measuring subjective states without relying on rating scales. The correlations to rating scales tend to be moderate for unipolar similarity scales and strong for bipolar similarity scales (Table 10, Figure 3). With regard to the satisfaction words, the unipolar satisfaction semantic similarity scale correlates with the SWLS score (in Study 9 T2: $r=.33$ ), and the bipolar satisfaction semantic similarity scale correlates with the SWLS score to an even higher degree $(r=.60)$. Similarly, with regard to the harmony words, the unipolar harmony semantic similarity scale correlates with the HILS score $(r=.43)$, and the bipolar harmony semantic similarity scale yields a higher correlation ( $\mathrm{r}=.65)$.

However, it is noteworthy that the semantic similarity scales sometimes correlate higher to a rating scale it is not intended to measure compared with the intended target construct. That is, it could be expected that the semantic similarity scales generated for each construct would 
exhibit the highest correlation in relation to its specific rating scale. This is the case when it comes to the harmony words and the harmony semantic similarity scale, where the correlation is higher with regard to the HILS ( $\mathrm{r}=.43$ ) than with regard to the SWLS ( $\mathrm{r}=.34)$; however, when it comes to the satisfaction words and the satisfaction semantic similarity scale, there is a higher correlation with regard to the HILS (r=.35) and not the SWLS (r=.33). Similarly, with regard to the worry words and the worry semantic similarity scale, there is a higher correlation when it comes to the GAD-7 ( $\mathrm{r}=.39)$ than to the PHQ-9 $(\mathrm{r}=.32)$, whereas the depression words and the depression semantic similarity scale do not exhibit a higher correlation when it comes to the PHQ-9 ( $\mathrm{r}=.29)$, but rather when it comes to the GAD-7 ( $\mathrm{r}=.31)$. Hence, there is a lack of clear target specificity among semantic similarity scales and rating scales.

Table 10

Word-Norms Measure Constructs Independently from Rating Scales as Seen by their InterCorrelations

\begin{tabular}{|c|c|c|c|c|c|c|c|c|c|c|c|c|c|}
\hline \multirow{2}{*}{$\begin{array}{l}\text { Measure Type } \\
\text { Measure }\end{array}$} & \multirow[b]{2}{*}{1} & \multicolumn{3}{|c|}{ Numeric scales } & \multirow[b]{2}{*}{5} & \multicolumn{3}{|c|}{ Valence } & \multirow[b]{2}{*}{9} & \multicolumn{4}{|c|}{ SSS } \\
\hline & & 2 & 3 & 4 & & 6 & 7 & 8 & & 10 & 11 & 12 & 13 \\
\hline 1. HILS & & & & & & & & & & & & & \\
\hline 2. SWLS & .83 & & & & & & & & & & & & \\
\hline 3. GAD-7 & -.67 & -.60 & & & & & & & & & & & \\
\hline 4. PHQ-9 & -.67 & -.64 & .86 & & & & & & & & & & \\
\hline 5. Hw: Valence & .74 & .59 & -.55 & -.53 & & & & & & & & & \\
\hline 6. Sw: Valence & .71 & .67 & -.51 & -.52 & .70 & & & & & & & & \\
\hline 7. Ww: Valence & .52 & .48 & -.61 & -.52 & .45 & .48 & & & & & & & \\
\hline 8. Dw: Valence & .59 & .55 & -.60 & -.62 & .50 & .51 & .72 & & & & & & \\
\hline 9. Hw: Bipolar & .65 & .52 & -.52 & -.49 & .87 & .64 & .42 & .44 & & & & & \\
\hline 10. Sw: Bipolar & .64 & .60 & -.47 & -.47 & .63 & .89 & .44 & .45 & .62 & & & & \\
\hline 11. Hw: Unipolar & .43 & .34 & -.38 & -.36 & .57 & .43 & .33 & .33 & .72 & .46 & & & \\
\hline 12. Sw: Unipolar & .35 & .33 & -.28 & -.30 & .32 & .51 & .27 & .23 & .36 & .63 & .44 & & \\
\hline 13. Ww: Unipolar & -.32 & -.29 & .39 & .32 & -.34 & -.37 & -.52 & -.37 & -.33 & -.39 & -.21 & $-.12 * *$ & \\
\hline 14. Dw: Unipolar & -.30 & -.28 & .31 & .29 & -.27 & -.29 & -.31 & -.33 & -.26 & -.28 & -.20 & ns & .60 \\
\hline
\end{tabular}

Note. Pearson's r correlations, all correlations were significant at $\mathrm{p}<.001$ unless otherwise specified, $* *=\mathrm{p}<.01, \mathrm{~ns}=\mathrm{not}$ significant. N=477. HILS=Harmony in Life Scales; SWLS=Satisfaction with Life Scale; GAD-7=Generalized Anxiety Disorder-7; PHQ-9=Patient Health Qustionnaire-9; Hw=Harmony words; Sw=Satisfaction words; $\mathrm{Ww}=$ Worry words; $\mathrm{Dw}=$ Depression words; Valence=Semantic Predicted Valence Scales; Bipolar=Bipolar Semantic Similarity Scale; Unipolar=Unipolar Semantic Similarity Scale. 


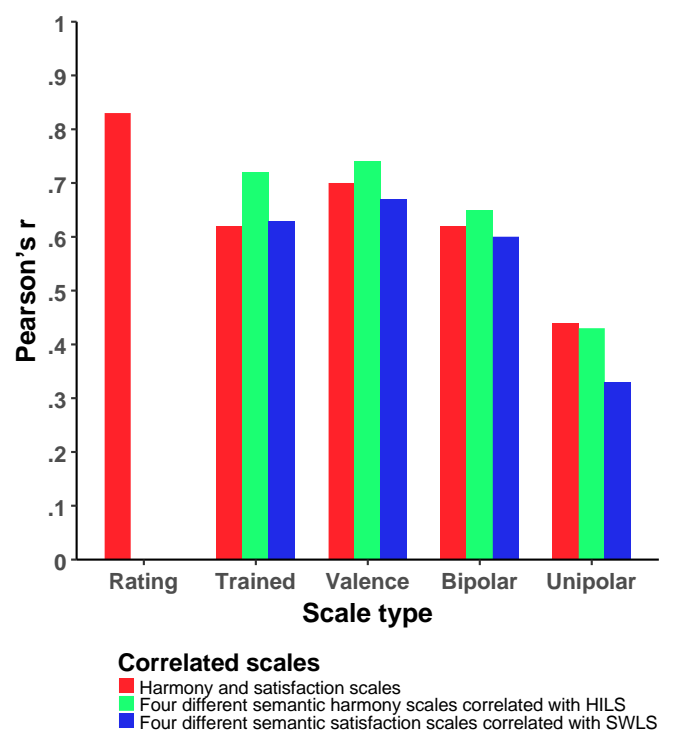

Figure 3. Pearson correlation (y-axis) among the various types of scales (x-axis) for harmony and satisfaction. The red bars show the correlation between harmony and satisfaction for one numerical measure (HILS-SWLS) and for four different semantic measures. The green bars show HILS correlated with four different semantic measures of harmony, while the blue bars similarly show corresponding correlations for SWLS and four semantic measures of satisfaction. Rating $=$ numerical rating scales; Trained $=$ trained predicted scales between word responses and rating scales; Valence $=$ semantic predicted ANEW valence scales; Bipolar = bipolar semantic similarity scales; Unipolar = unipolar semantic similarity scales.

\section{Semantic Similarity Scales Differentiate between Similar Constructs}

We suggest that the less than perfect correlations of semantic similarity scales to rating scales may not necessarily indicate a measurement error in the semantic measures. Instead, semantic similarity scales may measure different qualities that efficiently differentiate between similar constructs, whereas rating scales tend to capture one-dimensional valence. This suggestion was tested by applying an independently trained model of the ANEW (Bradley \& Lang, 1999) in order to create semantic predicted valence scales. According to our suggestion, these semantic valence scales correlate significantly stronger with respective rating scales compared with the bipolar similarity scales $(z=4.32-5.61, p<.001$, two-tailed; Lee \& Preacher, 2013). Further, using the valence scale as a covariate when training semantic responses to rating scales reduces 
the correlation considerably (Table 6). Hence, rating scales are highly influenced by general valence; potentially driven by a general positive or negative attitude to life, rather than distinct answers related to targeted constructs. This interpretation is consistent with findings that the rating scales have strong intercorrelations (see also Kashdan, Biswas-Diener \& King, 2008). On the other hand, the similarity scales based on construct-specific word norms exhibit a lower intercorrelation compared with rating scales. In addition, semantic $t$-tests further support the semantic difference between semantic responses by discriminating well between the semantic responses with medium to large effect sizes (Table 11). This suggests that semantic measures more clearly tap into the targeted construct, which is supported further when describing the constructs using word plots. 
Table 11

Semantic Questions Responses Differ Significantly between Related Psychological Constructs Harmony vs. Satisfaction words

Depression vs. Worry words

\begin{tabular}{llllllllllll}
\hline Study: Condition & S3 & S4 & S5 & S6 & S7: 1-w & S7: 3-w & S7: 5-w & S9: T1 & S9: T2 & S8 & S9:T2 \\
\hline t-value & 2.67 & 12.71 & 10.75 & 7.70 & 12.36 & 15.56 & 12.10 & 23.66 & 18.08 & 16.86 & 18.43 \\
Cohen's d & .28 & .73 & .63 & .56 & .66 & .83 & .76 & .81 & .85 & .85 & .84 \\
\hline
\end{tabular}

Note. This table presents semantic t-tests of the summarized semantic representations of the related psychological constructs. S=Study; $\mathrm{w}=$ words; T=Time; $\mathrm{S} 3$ was significant at $p<.01$, all others at $\mathrm{p}<.001$; where all except $\mathrm{S} 3$, encompass medium to large effect sizes 


\section{Describing Constructs with Keywords}

Figures 4-6 provide empirically derived depictions of the studied constructs by plotting words that significantly discriminate in relevant dimensions, including different semantic responses (x-axes) and semantic scales or rating scales (y-axes). The plots tend to conceptualize the constructs in a meaningful way, which is also intuitively consistent with a theoretical understanding of the constructs as outlined in the introduction. The semantic responses concerning satisfaction with life highlight words such as happy, content and fulfilled, whereas harmony in life responses highlight peace, calm and balance. The semantic responses concerning depression and worry are also distinct, where depression is significantly related to words such as sad, down and lonely, and worry is associated with anxious, nervous and tense. This supports the construct validity of the semantic measures. Semantic measures empirically describe/define the constructs.

The ability of measures to offer a good differentiation between constructs may be tested further by examining the number of significant words and their position in the figures when covarying the different scales. Scales that are independent should still yield significant words on the axis where they are being covaried (here the y-axis). For example, if the semantic similarity scales of harmony and satisfaction measure the respectively targeted construct with high independence, it should follow that covarying the scales with each other would impact the plotted harmony and satisfaction word responses differently compared with their original positions without a covariate. In contrast, if the scales are not measuring the constructs differently/independently, the impact of covarying the scales would have a similar effect on the position of both the harmony and the satisfaction word responses, where both sets of words would be positioned to not be significant on the y-axis. That is, for semantic similarity scales we hypothesize an independence between the word responses, in which such independence may manifest itself in such a way that word responses for the targeted construct are positioned 
on the higher end of the y-axis, whereas the word responses related to the covaried construct are positioned on the lower end of the y-axis. In contrast, when covarying the numerical rating scales, we hypothesize that word responses between constructs will not show independence, and thus not be significant on the y-axis anymore.

Indeed, the results show that covarying the harmony semantic similarity scale with the satisfaction semantic similarity scale (or vice versa) reveals a significant independence between these constructs by yielding correlations to relevant words describing the construct; i.e., 44 and 37 significant words on the $y$-axes in Figure $4 \mathrm{e}$ and $\mathrm{f}$, respectively. This is not the case when covarying the corresponding numerical rating scales; i.e., only 6 and 0 significant words on the $\mathrm{y}$-axes in Figure $4 \mathrm{~g}$ and $\mathrm{h}$, respectively. This independence is also found with regard to depression and worry (Figure 5). When the semantic similarity scales of worry and depression are covaried with each other, there are 35 and 36 significant words on the $y$-axes in Figure 5e and f, respectively. However, when the rating scales of depression and worry are covaried, there are only 6 and 22 significant words on the y-axes in Figure $5 \mathrm{~g}$ and $\mathrm{h}$, respectively.

Further, the semantic similarity scales actually tap into different aspects than the semantic predicted ANEW valence scales and the rating scales, which becomes clear when described in the word figures. The figures plotting rating scales and semantic predicted valence scales tend to reveal a similar structure, which suggests that rating scales focus on valence. Meanwhile, the semantic similarity scales demonstrate a structure that is more in line with their theoretical conceptualizations. This becomes even more clear when covarying the semantic predicted ANEW valence scales in figures. That is, figures with harmony and satisfaction semantic similarity scales covaried with the semantic predicted ANEW valence scale have 23 and 24 significant words in Figure $6 \mathrm{c}$ and d, respectively. Meanwhile, the harmony and satisfaction rating scales covaried with the semantic predicted ANEW valence scale reveal 0 
significant words (Figure 6 e-f). This is also the case when it comes to depression and worry: in terms of semantic similarity scales, 25 and 26 words are significant in Figure $6 \mathrm{~g}$ and $\mathrm{h}$, respectively, and in terms of rating scales, 0 words are significant (Figure $6 \mathrm{i}-\mathrm{j}$ ). It should be pointed out that this offers further support for the strong, one-dimensional link between rating scales and valence. Overall, these results suggest that similarity scales more clearly differentiate between the psychological constructs compared with rating scales. 

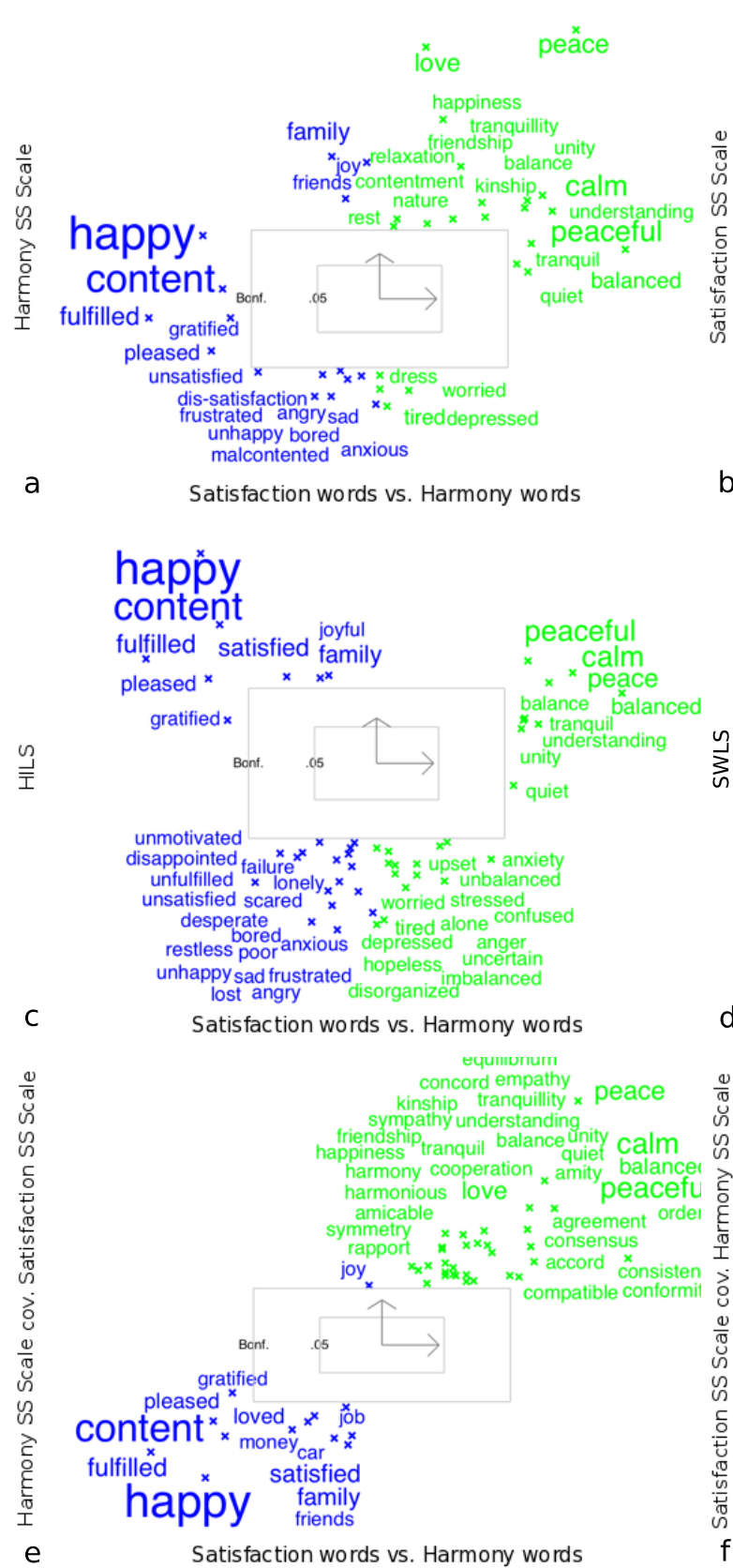

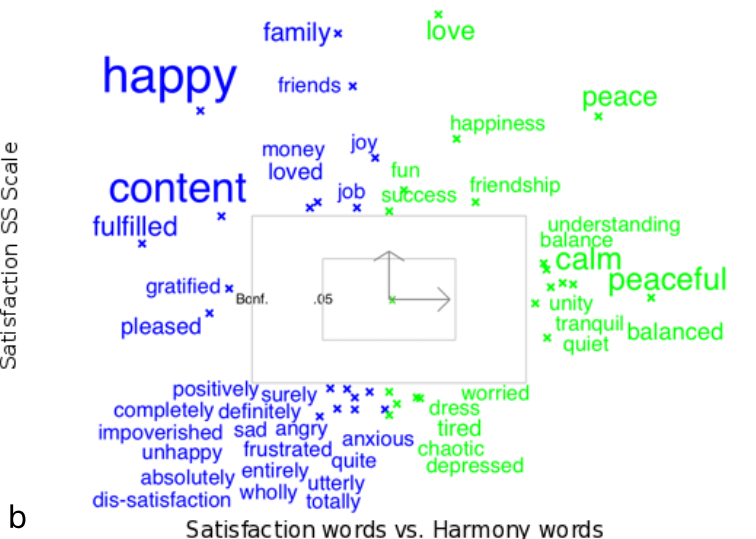

\section{happy \\ content family}

fulfilléd satisfied joyful

pleased $x$

gratified $x$

gratified $\mathbf{x}$

$x x$
peaceful $^{\text {banced }}$

\section{happy family fulfilled friends ${ }^{x} x$ Content $\underset{x}{\text { money }}$ loved $x$}

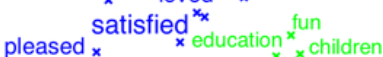
$\underset{\text { gratified } x}{x}$

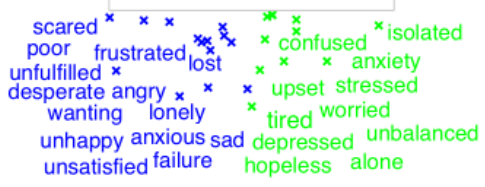

Satisfaction words vs. Harmony words

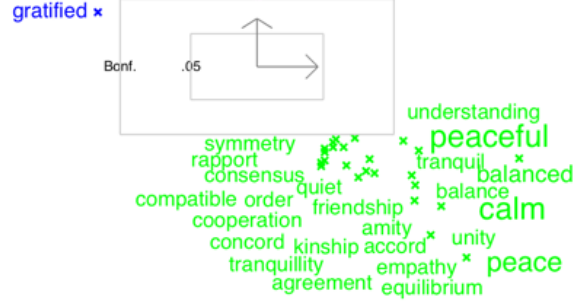

Satisfaction words vs. Harmony words

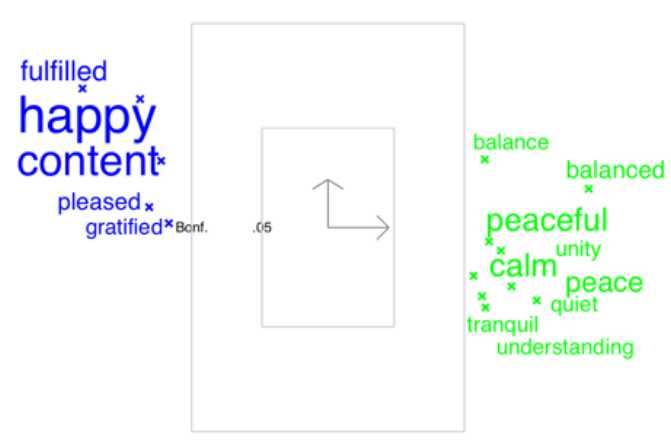

Satisfaction words vs. Harmony words 
Figure 4a-f. Semantic measures differentiate between constructs of satisfaction and harmony as shown by plotting significant keywords. The figures also show the independence of constructs using semantic measures (e, f) as compared with numerical rating scales $(\mathrm{g}, \mathrm{h})$. On the $\mathrm{x}$-axis, words are plotted according to the $\chi$-values from a Chi-square test, with a Bonferroni correction for multiple comparisons (Bonf.=Bonferroni line where $\chi=4.04, .05$ indicates the uncorrected $\mathrm{p}$-value line, where $\chi=1.96)$. On the $y$-axis, words are plotted according to point biserial correlations ( $\mathrm{r}=.13$ at the Bonferroni line, and $\mathrm{r}=.06$ at the. 05 uncorrected $p$-value line). More frequent words are plotted with a larger font size with fixed lower and upper limits. The $\mathrm{x}$-axes represent $\chi$-values associated with words generated in relation to satisfaction (blue/left) versus harmony (green/right). The y-axes show significant words related to Semantic Similarity $(\mathrm{SS})$ scales or rating scales; HILS = Harmony in Life Scale; SWLS = Satisfaction with Life Scale; cov = covariate. $N=477$. 


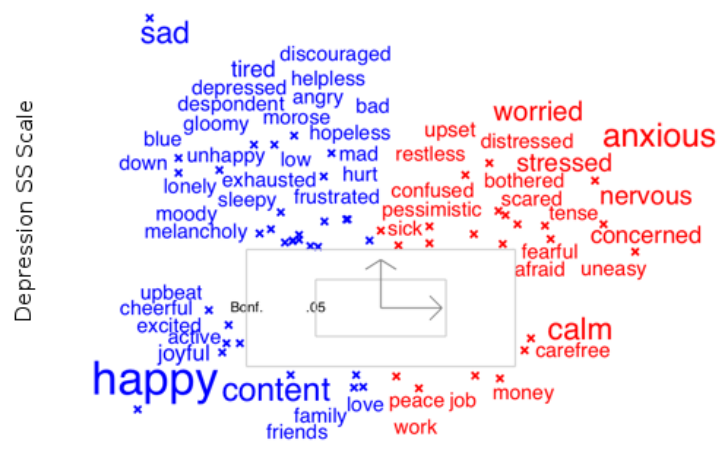

a

Depression words vs. Worry words

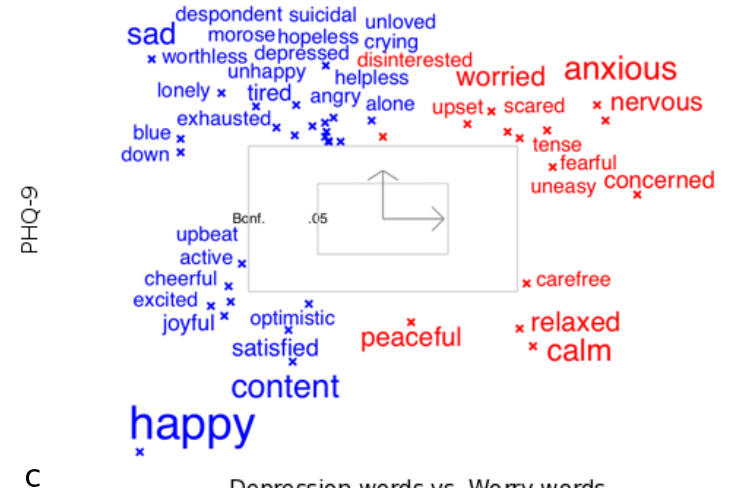

C

Depression words vs. Worry words b

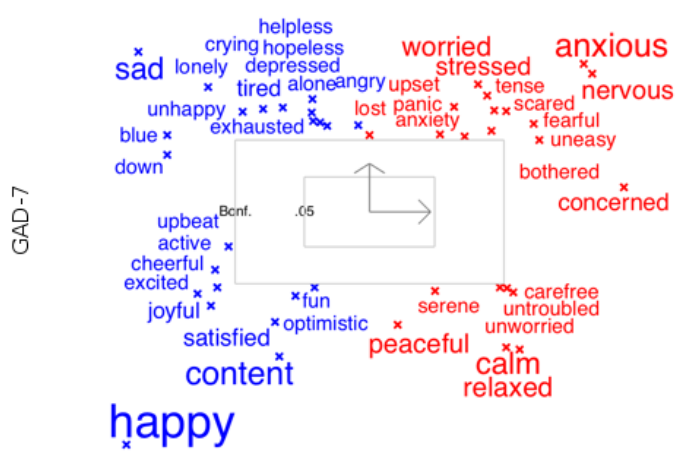

d

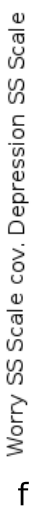

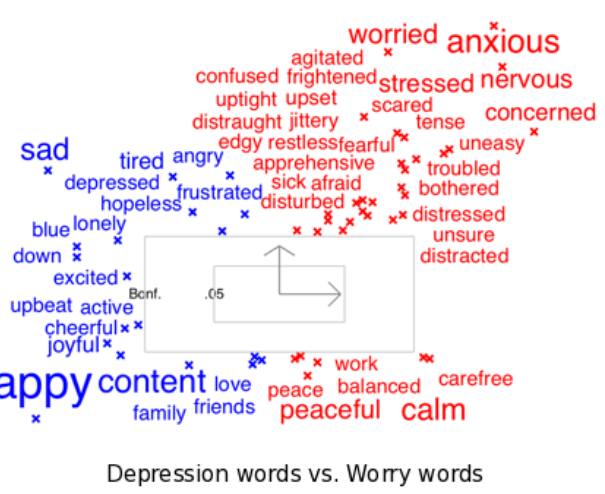

Depression words vs. Worry words

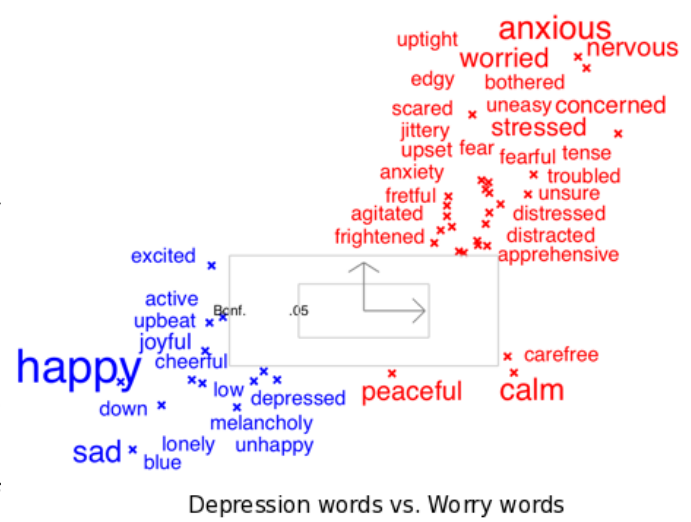

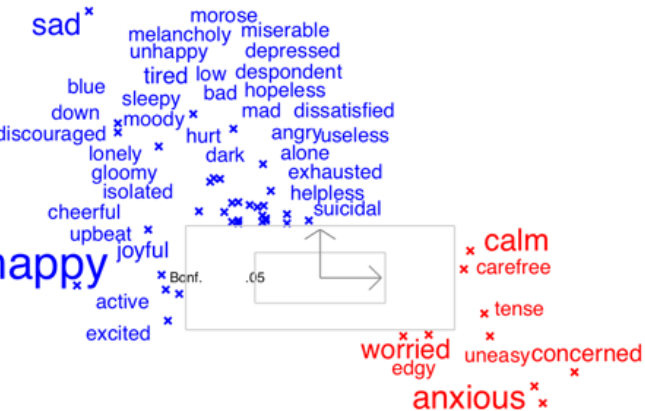

Depression words vs. Worry words

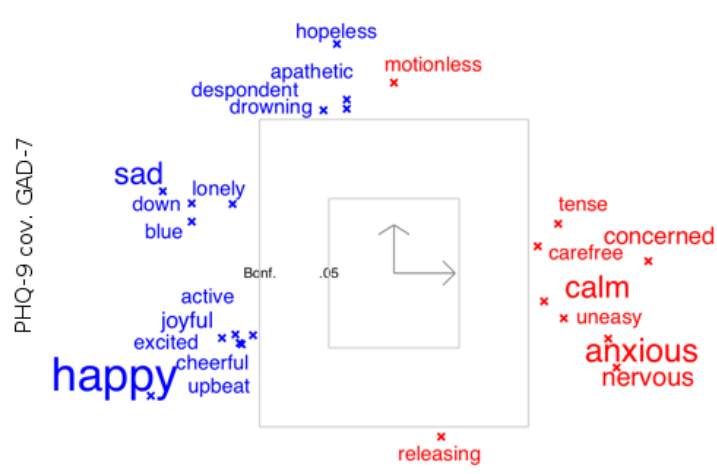

Depression words vs. Worry words

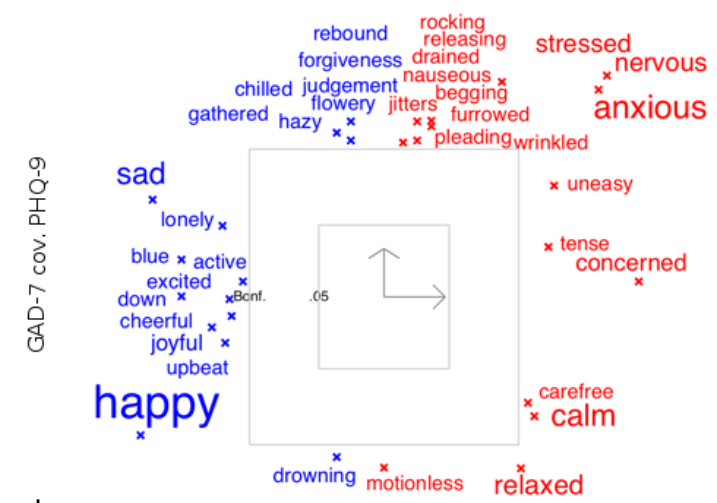

$\mathrm{h}$

Depression words vs. Worry words 
Figure $5 a$-h. Semantic measures differentiate between constructs of depression and worry as shown by plotting significant keywords. The figures also show the independence of constructs using semantic measures (e, f) as compared with numerical rating scales $(\mathrm{g}, \mathrm{h})$. On the $\mathrm{x}$-axis, words are plotted according to the $\chi$-values from a Chi-square test, with a Bonferroni correction for multiple comparisons (Bonf. $=$ Bonferroni line where $\chi=4.04$, .05 indicates the uncorrected $p$-value line, where $\chi=1.96$ ). On the $y$-axis, words are plotted according to pointbiserial correlations ( $r=.13$ at the Bonferroni line, and $r=.06$ at the .05 uncorrected p-value line). More frequent words are plotted with a larger font with fixed lower and upper limits. The $\mathrm{x}$-axes represent $\chi$-values associated with words generated in relation to depression (blue, left) versus worry (red, right). The y-axes show significant words related to Semantic Similarity (SS) scales or rating scales; PHQ-9 = Patient Health Questionnaire-9; GAD7 = Generalized Anxiety Disorder scale-7 (GAD-7); cov. = covariate; $N=477$. 


\section{happy}

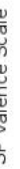

Satisfaction words vs. Harmony words

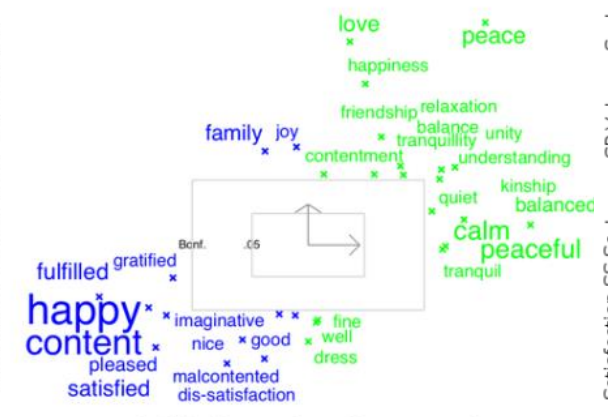

c

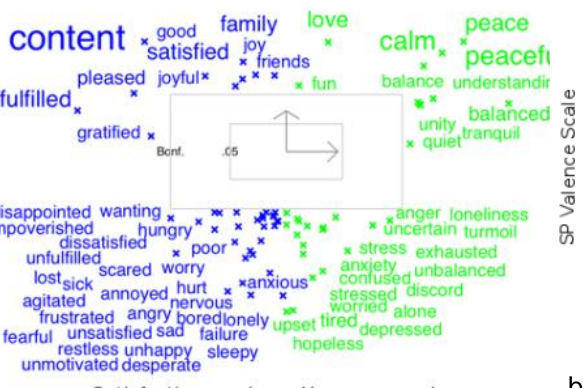

b

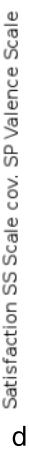

hầppy satistient $_{\text {conten }}$

joyful optimistic fun

cheerful joy loved peaceful harmony

pleased $x$
excied $x$
healthy

healthy friends $x \times$ free $x$ comfortable $x$ relaxed

encouraged ${ }_{\text {blestived }} x^{x} \times{ }^{x} x^{x} x^{x} \times$

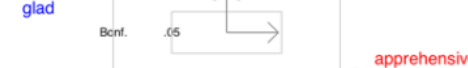

lonely
blue $x$ moody alone $x$

down $x$ hopeless $x_{x}$ restless stress $x_{x} x_{x} x^{*} x^{*}$ unsure $x^{x}$

sad unhappy frustrated worry $x$ a anxiety $x^{x}$ anxious tired angry sick afraid tense anxious
depressed
scared fear uneasy exhausted disturbed upset wored worried

frightened frotfiul fearful
pessimistic

Depression words vs. Worry words

$\underset{\text { friends }}{\text { family }^{x}} \stackrel{x}{x^{x}}$

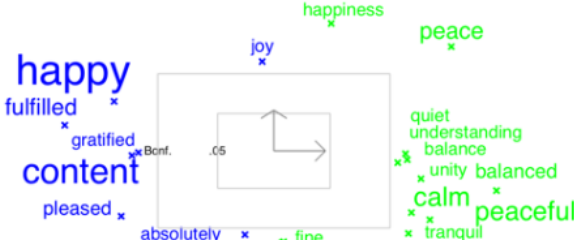

pleased $_{x}$

completely ${ }_{x}{ }_{x}^{x} *$ fin

utterly surely ${ }^{x}$ good

nice wholly

malcontented dis-satisfaction

Satisfaction words vs. Harmony words

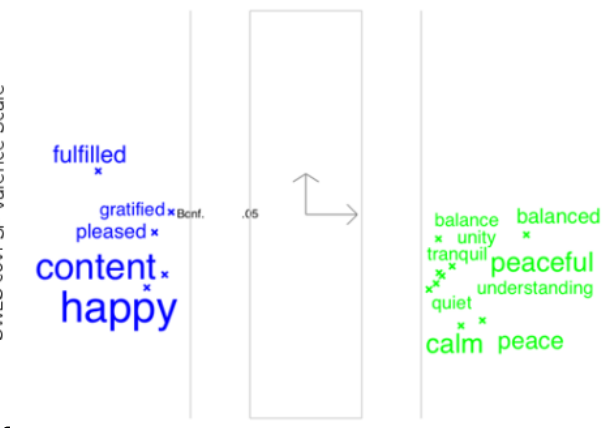

Satisfaction words vs. Harmony words

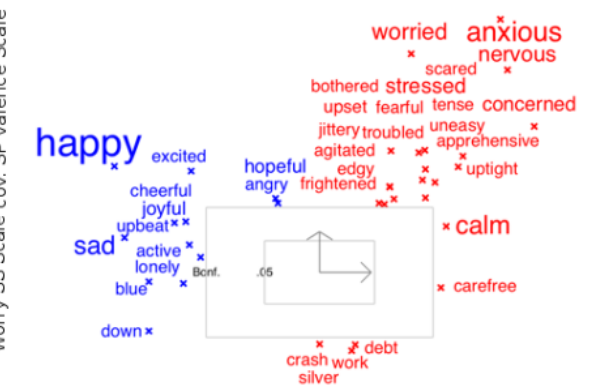

h

Depression words vs. Worry words

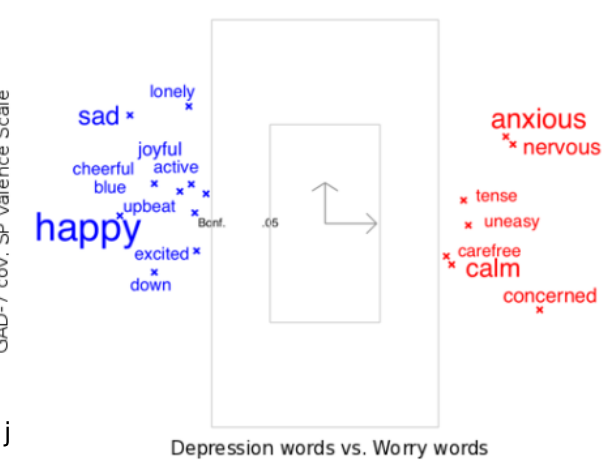


Figure $6 a$-j. Compared with rating scales, semantic measures do a better job differentiating between mental health constructs beyond valence. On the x-axis, words are plotted according to the $\chi$-values from a Chi-square test, with a Bonferroni correction for multiple comparisons (Bonf.=Bonferroni line where $\chi=4.04, .05$ indicates the uncorrected $\mathrm{p}$-value line, where $\chi=1.96)$. On the $\mathrm{y}$-axis, words are plotted according to point-biserial correlations $(\mathrm{r}=.13$ at the Bonferroni line, and $\mathrm{r}=.06$ at the .05 uncorrected $p$-value line). More frequent words are plotted with a larger font with fixed lower and upper limits. The x-axes represent $\chi$-values associated with words generated in relation to a, c-f) satisfaction (blue, left) versus harmony (green, right) and $b, g-j$ ) depression (blue, left) versus worry (red, right). The y-axes in Figures c-j are covaried (cov.) with the semantic predicted (SP) ANEW valence scale; SS=Semantic Similarity; HILS = Harmony in Life Scale; SWLS = Satisfaction with Life Scale; PHQ-9 = Patient Health Questionnaire-9; GAD-7 = Generalized Anxiety Disorder scale-7. N=477. 


\section{Test-Retest}

Semantic measures exhibit satisfactory test-retest reliability over 31 days (Table 12). Trained scales tend to demonstrate moderate correlations for both harmony in life and satisfaction with life. Although unipolar semantic similarity scales demonstrate low test-retest correlations, bipolar semantic similarity scales show moderate correlations for both harmony and satisfaction.

Table 12

Semantic Questions Demonstrate Satisfactory Test Re-Test Reliability for the Well-Being Measures in Study 9 Between T1 and T2

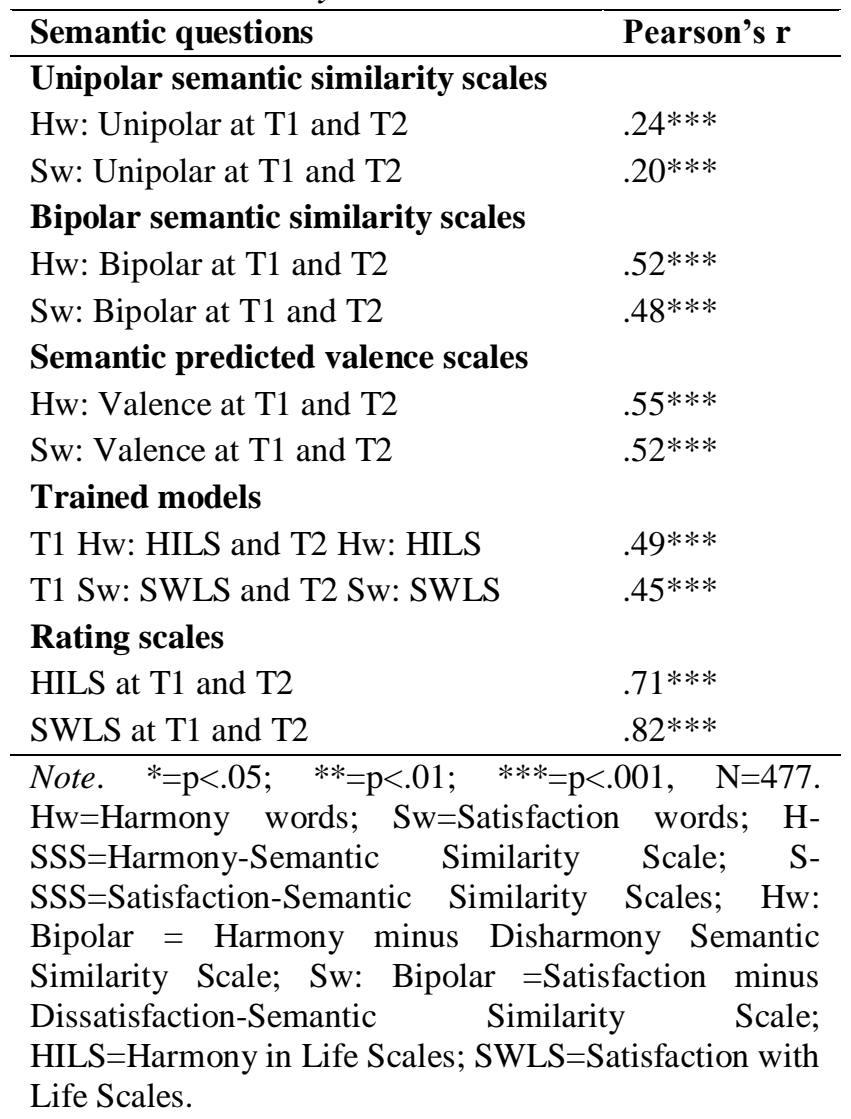




\section{Social Desirability}

Overall, the semantic measures, as compared with rating scales, are associated with less social desirability as measured using the Marlowe-Crowne Social Desirability Scale, the short version Form A (Table 13; Reynolds, 1982). The rating scales yield the anticipated positive correlations with the well-being measures and negative correlations with the ill-being measures. Training the semantic content to the social desirability scale only yielded a weak significant relationship for the semantic question of depression. The unipolar semantic similarity scales only yielded a low significant relationship between worry and social desirability. Meanwhile, among the bipolar semantic similarity scales, only harmonydisharmony displayed a weak significant relationship to social desirability. Compared with the rating scales, the semantic predicted ANEW valence scale displayed correlations with similar strength for the ill-being measures and somewhat weaker strength for the well-being measures. Note that all correlations between social desirability and the semantic predicted ANEW valence scale were positive, as positivity in response to the semantic questions tends to relate to social desirability. Further, plotting the words reveals that there are no words significantly related to low or high social desirability scores.

Table 13

Semantic Questions Show Lower Social Desirability than Rating Scales

\begin{tabular}{|c|c|c|c|c|c|c|}
\hline \multicolumn{2}{|c|}{$\begin{array}{l}\text { Correlated } \\
\text { Constructs }\end{array}$} & $\begin{array}{l}\text { Training } \\
\text { to MC- } \\
\text { SDS-FA }\end{array}$ & $\begin{array}{l}\text { Valence } \\
\text { SP scales }\end{array}$ & $\begin{array}{l}\text { Unipolar } \\
\text { SS scales }\end{array}$ & $\begin{array}{l}\text { Bipolar SS } \\
\text { scales }\end{array}$ & $\begin{array}{l}\text { Rating } \\
\text { scales }\end{array}$ \\
\hline \multirow{4}{*}{$\begin{array}{l}\text { MC- } \\
\text { SDS- } \\
\text { FA }\end{array}$} & Harmony & $\mathrm{ns}$ & $.11 *$ & ns & $.11 *$ & $.19 * * *$ \\
\hline & Satisfaction & ns & $.11 *$ & ns & ns & $.19 * * *$ \\
\hline & Worry & ns & $.15^{* * * *}$ & $-.10^{*}$ & $N A$ & $-.16^{* * *}$ \\
\hline & Depression & $.08^{*}$ & $.13 * *$ & $\mathrm{~ns}$ & $N A$ & $-.14 * *$ \\
\hline
\end{tabular}




\section{Discussion on Reports regarding Subjective States}

The results reveal that semantic measures may be used for measuring, differentiating and describing subjective experiences of psychological constructs, including both well-being and ill-being. It is demonstrated that trained semantic responses predict rating scales scores with a high level of accuracy; which hold throughout seven studies involving differences in terms of required word responses (with 1, 3, 5, or 10 descriptive words, or free text as presented in the SM) and varying levels of detailed instructions. In addition, using semantic similarity scales enables measuring, differentiating and describing psychological constructs independent from rating scales. It is also shown that semantic measures show satisfactory test-retest reliability. Further, the four semantic questions, as compared with the corresponding rating scales, appear less susceptible to social desirability. Arguably, this might be because they promote a more personal and thus honest account of one's state of mind.

\section{Semantic Measures versus Rating Scales}

In our studies, semantic similarity scales appear to exhibit higher construct specificity and independence to similar constructs compared with rating scales. The word figures exhibit target specificity and independence when it comes to semantic similarity scales but not rating scales, even though there is a lack of target specificity among correlations between semantic similarity scales and respective rating scales. In addition, this target specificity and independence were found for both descriptive words in relation to harmony, satisfaction, depression and worry, as well as text responses in relation to harmony and satisfaction (see SM). Overall, the results support that semantic measures demonstrate high construct validity where they clearly tap into the to-be-measured construct, whereas rating scales to a greater extent appear to be tapping into a common construct relating to valence.

Valence-Focused Rating Scales and Construct-Specific Semantic Measures. What rating scales might have in common is that they more strongly tap into valence, whereas 
semantic similarity scales are better at differentiating between constructs. The hypothesis that rating scales are highly valence-driven is supported empirically by the results showing that covarying the semantic predicted ANEW valence scales when training the semantic responses to rating scales largely reduces the correlations. Similarly, our results show that the semantic predicted ANEW valence scales of each semantic response have a higher correlation with the rating scales as compared with respective semantic similarity scale. Hence, the affective valence of the generated words is strongly related to the rating scales.

In addition, the word figures demonstrate that semantic similarity scales, as compared with rating scales, are more distinct from the semantic predicted ANEW valence scales. That rating scales exhibit none or few significant words when being covaried with the semantic predicted ANEW valence scales, as well as with each other, is also in accordance with previous findings, including the high intercorrelation among rating scales and the lack of target specificity between correlations of rating scales and semantic similarity scales.

From a theoretical perspective, we argue that rating scales and valence have onedimensionality in common. That is, valence is conceptualized as ranging from pleasant to unpleasant (i.e., in the construction of the ANEW, words were rated on a picture-based scale depicting pictures of happy, smiling to unhappy, frowning characters; Bradley \& Lang, 1999) This is arguably similar to the one-dimensional response format often used for rating scales. Response formats for rating scales tend to focus on the respondent's positive or negative stance on various statements (although the scale may differ, such as ranging from strongly disagree to strongly agree or not at all to nearly every day). This may potentially explain the lack of clear specificity among rating scales and semantic similarity scales.

Overall, the results suggest that rating scales are more focused on valence compared with semantic similarity scales, whereas semantic similarity scales are more focused on the tobe-measured construct compared with rating scales. Hence, the results indicate that semantic 
similarity scales, independent from rating scales, may be used for measuring, differentiating and describing participants' subjective experience of harmony in life, satisfaction with life, depression and worry with high validity and reliability.

\section{Limitations, Future Research and Overall Conclusions}

Answering semantic questions seems to require more time and effort for participants compared with rating scales. In the studies on reports regarding external stimuli, the semantic questions conditions, as compared with the rating scales conditions, took longer to complete for participants (see Table 2). In terms of effort, the semantic questions conditions yield lower percentages of participants completing the study, presumable due to the effort required for generating semantic answers. Even though the randomization procedure should divide $50 \%$ of the participants in the semantic questions condition, there were only $45 \%$ in Study 1 and $42 \%$ in Study 2 completing this condition. However, it should be noted that semantic questions conditions may be rendered less time-consuming by requesting fewer words; for example, we found that it is possible to achieve good results by only requiring one word, as well as using short instructions in reports regarding subjective states. In addition, the relatively higher level of effort needed for semantic questions might be one of the strengths of this approach. That is, semantic questions may lead to more thought through answers. In contrast, instructions for rating scales often ask respondents to not think for too long on each question, but instead answer with what first comes to mind.

The current studies focus on psychological constructs relating to well-being and mental health problems; however, future studies could study a broader range of psychological constructs and contexts. Further, the current semantic questions allow the respondent to decide for him- or herself what is important for the to-be-measured construct. However, in terms of mental health diagnoses, future research could examine to what extent these semantic questions cover important diagnostic criteria outlined in manuals such as the DSM-5. 
Moreover, the studies on subjective states only include self-report rating scales, whereas future studies should evaluate these using objective measures. In addition, whereas the current studies compare semantic measures with rating scale methodologies, future studies may also compare them with interview techniques (e.g., the International Neuropsychiatric Interview; MINI; Sheehan et al., 1998). Finally, future studies could also explore potential advantages with using semantic representations based on other algorithms, such as COALS, as well as word frequency strategies, such as LIWC.

To sum up, our results from experiments based on both external stimuli and subjective states show that semantic measures are competitive, or better, compared with numerical rating scales when evaluated both in terms of validity and reliability. Semantic measures address limitations associated with rating scales, for example by allowing unrestricted open-ended responses. Importantly, semantic similarity scales enable measuring constructs independent of rating scales, and we demonstrated that they are very good at differentiating even between similar constructs. Trained semantic scales may also be used for closely studying the relationship between texts/words and numeric values. This is particularly valuable when numeric values represent objective outcomes rather than subjective rating scales, when studying a particular characteristic of a word response, such as valence, or in situations when it is difficult to construct a representative word norm (such as in the case of social desirability). Future research should investigate to what extent these results generalize to other psychological constructs, situations and contexts.

That semantic measures are able to measure, differentiate and describe psychological constructs in two different paradigms demonstrate their potential to improve the way we quantify individual states of mind, and thus our understanding of the human mind. Therefore, we argue that semantic measures offer an alternative method for quantifying mental states in research and professional contexts (e.g., surveys, opinion polls, job recruitment, etc.). 


\section{References}

American Psychiatric Association. (2013). Diagnostic and statistical manual of mental disorders (5th ed.). Washington, D.C: American Psychiatric Association.

Arvidsson, D., Sikström, S., \& Werbart, A. (2011). Changes in self and object representations following psychotherapy measured by a theory-free, computational, semantic space method. Psychotherapy Research, 21(4), 430-446. doi:10.1080/10503307.2011.577824

Axelson, D. A., \& Birmaher, B. (2001). Relation between anxiety and depressive disorders in childhood and adolescence. Depression and Anxiety, 14(2), 67-78. doi:10.1002/da.1048

Bartlett, M. S., Viola, P. A., Sejnowski, T. J., Golomb, B. A., Larsen, J., Hager, J. C., \& Ekman, P. (1996). Classifying facial action. Advances in neural information processing systems, 823-829.

Bradley, M. M., \& Lang, P. J. (1999). Affective norms for English words (ANEW): Instruction manual and affective ratings. Retrieved from

Brown, T. A. (2007). Temporal course and structural relationships among dimensions of temperament and DSM-IV anxiety and mood disorder constructs. Journal of Abnormal Psychology, 116(2), 313-328. doi:10.1037/0021-843X.116.2.313

Brown, T. A., Chorpita, B. F., \& Barlow, D. H. (1998). Structural relationships among dimensions of the DSM-IV anxiety and mood disorders and dimensions of negative affect, positive affect, and autonomic arousal. Journal of Abnormal Psychology, 107(2), 179-192. doi:10.1037/0021-843X.107.2.179

Buhrmester, M., Kwang, T., \& Gosling, S. D. (2011). Amazon's Mechanical Turk: A new source of inexpensive, yet high-quality, data? Perspectives on Psychological Science, 6(1), 3-5. doi:10.1177/1745691610393980 
Crowne, D. P., \& Marlowe, D. (1960). A new scale of social desirability independent of psychopathology. Journal of Consulting Psychology, 24(4), 349-354. doi:10.1037/h0047358

Diener, E., Emmons, R. A., Larsen, R. J., \& Griffin, S. (1985). The satisfaction with life scale. Journal of Personality Assessment, 49(1), 71-75.

Dunn, T. J., Baguley, T., \& Brunsden, V. (2014). From alpha to omega: A practical solution to the pervasive problem of internal consistency estimation. British Journal of Psychology, 105(3), 399-412. doi:10.1111/bjop.12046

Ekman, P. (1992). Are there basic emotions? Psychological Review, 99(3), 550-553.

Foltz, P. W., Kintsch, W., \& Landauer, T. K. (1998). The measurement of textual coherence with latent semantic analysis. Discourse Processes, 25(2-3), 285-307.

Garcia, D., \& Sikström, S. (2013). Quantifying the Semantic Representations of Adolescents' Memories of Positive and Negative Life Events. Journal of Happiness Studies, 14(4), 1309-1323. doi:10.1007/s10902-012-9385-8

Gustafsson Sendén, M., Sikström, S., \& Lindholm, T. (2015). 'She' and 'He' in news media messages: Pronoun use reflects gender biases in semantic contexts. Sex Roles, 72(12), 40-49. doi:10.1007/s11199-014-0437-X

Gustafsson Sendén, M., Lindholm, T., \& Sikström, S. (2014). Biases in news media as reflected by personal pronouns in evaluative contexts. Social Psychology.

Golub, G., \& Kahan, W. (1965). Calculating the singular values and pseudo-inverse of a matrix. Journal of the Society for Industrial and Applied Mathematics, Series B: Numerical Analysis, 2(2), 205-224.

Karlsson, K., Sikström, S., \& Willander, J. (2013). The Semantic Representation of Event Information Depends on the Cue Modality: An Instance of Meaning-Based Retrieval. PLoS ONE, 8(10), 1-8. doi:10.1371/journal.pone.0073378 
Kashdan, T. B., Biswas-Diener, R., \& King, L. A. (2008). Reconsidering happiness: the costs of distinguishing between hedonics and eudaimonia. Journal of Positive Psychology, 3(4), 219-233. doi:10.1080/17439760802303044

Kern, M. L., Park, G., Eichstaedt, J. C., Schwartz, H. A., Sap, M., Smith, L. K., \& Ungar, L. H. (2016). Gaining Insights From Social Media Language: Methodologies and Challenges. Psychological Methods. Advance online publication. http://dx.doi.org/10.1037/met0000091

Kessler, R. C., Chiu, W., Demler, O., Merikangas, K. R., \& Walters, E. E. (2005). Prevalence, severity, and comorbidity of 12-month DSM-IV disorders in the National Comorbidity Survey Replication. Archives of General Psychiatry, 62(6), 617-627. doi:10.1001/archpsyc.62.6.617

Kjell, O. N. E. (2011). Sustainable Well-Being: A Potential Synergy Between Sustainability and Well-Being Research. Review of General Psychology, 15(3), 255-266. doi:10.1037/a0024603

Kjell, O. N. E., Daukantaitė, D., Hefferon, K., \& Sikström, S. (2015). The harmony in life scale complements the satisfaction with life scale: Expanding the conceptualization of the cognitive component of subjective well-being. Social Indicators Research. doi:10.1007/s11205-015-0903-Z

Kroenke, K., \& Spitzer, R. L. (2002). The PHQ-9: a new depression diagnostic and severity measure. Psychiatr Ann, 32(9), 1-7.

Kroenke, K., Spitzer, R. L., \& Williams, J. B. W. (2001). The PHQ-9: Validity of a Brief Depression Severity Measure. Journal of General Internal Medicine, 16(9), 606-613. doi:10.1046/j.1525-1497.2001.016009606.x

Krosnick, J. A. (1999). Survey research. Annual Review of Psychology, 50, 537-567. doi:10.1146/annurev.psych.50.1.537 
Kwantes, P. J., Derbentseva, N., Lam, Q., Vartanian, O., \& Marmurek, H. H. C. (2016). Assessing the Big Five personality traits with latent semantic analysis. Personality and Individual Differences, 102, 229-233.

doi:https://doi.org/10.1016/j.paid.2016.07.010

Landauer, T. K. (1999). Latent semantic analysis: A theory of the psychology of language and mind. Discourse Processes, 27(3), 303-310.

Landauer, T. K., \& Dumais, S. T. (1997). A solution to Plato's problem: The latent semantic analysis theory of acquisition, induction, and representation of knowledge. Psychological Review, 104(2), 211-240. doi:10.1037/0033-295x.104.2.211

Landauer, T. K., Foltz, P. W., \& Laham, D. (1998). An Introduction to Latent Semantic Analysis. Discourse Processes, 25(2-3), 259-284.

Landauer, T. K., McNamara, D., Dennis, S., \& Kintsch, W. (2007). Handbook of latent semantic analysis. University of Colorado Institute of cognitive science series: Lawrence Erlbaum Associates.

Langner, O., Dotsch, R., Bijlstra, G., Wigboldus, D. H., Hawk, S. T., \& van Knippenberg, A. (2010). Presentation and validation of the Radboud Faces Database. Cognition and Emotion, 24(8), 1377-1388.

Lee, I., \& Preacher, K. (2013). Calculation for the test of the difference between two dependent correlations with one variable in common [Computer software]. Retrieved January, 7, 2015.

Li, C. (2008). The philosophy of harmony in classical confucianism. Philosophy Compass, 3(3), 423-435.

Likert, R. (1932). A technique for the measurement of attitudes. Archives of Psychology, 22 140,55 . 
Löwe, B., Decker, O., Müller, S., Brähler, E., Schellberg, D., Herzog, W., \& Herzberg, P. Y. (2008). Validation and standardization of the Generalized Anxiety Disorder Screener (GAD-7) in the general population. Medical care, 46(3), 266-274.

Martin, A., Rief, W., Klaiberg, A., \& Braehler, E. (2006). Validity of the brief patient health questionnaire mood scale (PHQ-9) in the general population. General hospital psychiatry, 28(1), 71-77.

McNamara, D. S. (2011). Computational Methods to Extract Meaning From Text and Advance Theories of Human Cognition. Topics in Cognitive Science, 3(1), 3-17. doi:10.1111/j.1756-8765.2010.01117.x

Muntingh, A. D. T., van der Feltz-Cornelis, C. M., van Marwijk, H. W. J., Spinhoven, P., Penninx, B. W. J. H., \& van Balkom, A. J. L. M. (2011). Is the beck anxiety inventory a good tool to assess the severity of anxiety? A primary care study in The Netherlands study of depression and anxiety (NESDA). BMC Family Practice, 12, 66-66. doi:10.1186/1471-2296-12-66

Nakov, P., Popova, A., \& Mateev, P. (2001). Weight functions impact on LSA performance. EuroConference RANLP, 187-193.

Neuman, Y., \& Cohen, Y. (2014). A vectorial semantics approach to personality assessment. Scientific reports, 4.

Open Science Collaboration (2015). Estimating the reproducibility of psychological science. Science, 349(6251), aac4716.

Oppenheimer, D. M., Meyvis, T., \& Davidenko, N. (2009). Instructional manipulation checks: Detecting satisficing to increase statistical power. Journal of Experimental Social Psychology, 45(4), 867-872. doi:10.1016/j.jesp.2009.03.009

Park, G., Schwartz, H. A., Eichstaedt, J. C., Kern, M. L., Kosinski, M., Stillwell, D. J., . . Seligman, M. E. P. (2014). Automatic Personality Assessment Through Social Media 
Language. Journal of Personality and Social Psychology. doi:10.1037/pspp0000020 10.1037/pspp0000020.supp (Supplemental)

Pennebaker, J. W., Boyd, R. L., Jordan, K., \& Blackburn, K. (2015). The development and psychometric properties of LIWC2015. UT Faculty/Researcher Works.

Pennebaker, J. W., Francis, M. E., \& Booth, R. J. (2001). Linguistic inquiry and word count: LIWC 2001. Mahway: Lawrence Erlbaum Associates, 71, 2001.

Pennebaker, J. W., Mehl, M. R., \& Niederhoffer, K. G. (2003). Psychological aspects of natural language use: Our words, our selves. Annual Review of Psychology, 54(1), 547.

Reynolds, W. M. (1982). Development of reliable and valid short forms of the MarloweCrowne social desirability scale. Journal of Clinical Psychology, 38(1), 119-125. doi:10.1002/1097-4679(198201)38:1<119::aid-jclp2270380118>3.0.co;2-i

Rohde, D. L. T., Gonnerman, L. M., \& Plaut, D. C. (2005). An improved model of semantic similarity based on lexical co-occurrence. Unpublished manuscript. Retrieved from http://tedlab.mit.edu/ dr/Papers/RohdeGonnermanPlaut-COALS.pdf. Accessed 25 Sep 2017.

Roll, M., Mårtensson, F., Sikström, S., Apt, P., Arnling-Bååth, R., \& Horne, M. (2012). Atypical associations to abstract words in Broca's aphasia. Cortex, 48(8), 1068-1072. doi:10.1016/j.cortex.2011.11.009

Sarwar, F., Sikström, S., Allwood, C. M., \& Innes-Ker, Å. (2015). Predicting correctness of eyewitness statements using the semantic evaluation method (SEM). Quality \& Quantity, 49(4), 1735-1745.

Sheehan, D. V., Lecrubier, Y., Sheehan, K. H., Amorim, P., Janavs, J., Weiller, E., . . . Dunbar, G. C. (1998). The Mini-International Neuropsychiatric Interview (MINI): the 
development and validation of a structured diagnostic psychiatric interview for DSMIV and ICD-10. Journal of Clinical Psychiatry, 59, 22-33

Sinclair, S. J., Siefert, C. J., Slavin-Mulford, J. M., Stein, M. B., Renna, M., \& Blais, M. A. (2012). Psychometric evaluation and normative data for the Depression, Anxiety, and Stress Scales-21 (DASS-21) in a nonclinical sample of U.S. adults. Evaluation \& the Health Professions, 35(3), 259-279. doi:10.1177/0163278711424282

Spitzer, R. L., Kroenke, K., Williams, J. W. B., \& Löwe, B. (2006). A brief measure for assessing generalized anxiety disorder: The gad-7. Archives of Internal Medicine, 166(10), 1092-1097. doi:10.1001/archinte.166.10.1092

Watson, D., Clark, L. A., \& Carey, G. (1988). Positive and negative affectivity and their relation to anxiety and depressive disorders. Journal of Abnormal Psychology, 97(3), 346-353.

Wittchen, H. U., Kessler, R. C., Beesdo, K., Krause, P., Hofler, M., \& Hoyer, J. (2002). Generalized anxiety and depression in primary care: Prevalence, recognition, and management. Journal of Clinical Psychiatry, 63, 24-34. 


\section{Supplementary Material}

\section{Supplementary Material for Reports regarding External Stimuli \\ Results of Study 1}

Descriptive data of the number of words generated by participants in the semantic questions in relation to each facial expression are presented in Table S1. In the sematic questions condition, $81.5 \%$ of the facial expressions were categorized correctly with semantic predicted scales based on the leave-10\%-out procedure grouped according to participants. Using pictures as the grouping variable in the leave-10\%-out procedure yielded an overall correct categorization of $78.8 \%$. Meanwhile participants in the rating scales condition categorized $66.8 \%$ of the facial expressions correctly using the nine categorical alternatives. Hence, grouping according to participants $\left(\chi^{2}(1)=130.25, p<.001\right)$ or pictures $\left(\chi^{2}(1)=84.75, p<.001\right)$ yields significantly higher levels of overall correct categorizations compared with using rating scales. Grouping according to participants allows generalizing over different individuals, while grouping according to pictures allows generalizing to another set of pictures. Considering the somewhat lower level of overall correct categorizations when grouping according to picture, one should thus be more careful in terms of generalizing in relation to another set of pictures.

When using the unipolar semantic similarity scales, the overall correctness was significantly higher compared with the rating scales condition $\left(\chi^{2}(1)=84.75, p<.001\right)$, reaching 78.8\%. In addition, compared with the rating scales, using the bipolar semantic similarity scales also yielded a significantly higher $\left(\chi^{2}(1)=85.50, p<.001\right)$ overall correctness of $78.9 \%$. 
Table S1

Number of Generated and Unique Words in Reports Regarding Objective Stimuli

\begin{tabular}{llllllll}
\hline \multirow{2}{*}{ Study } & \multicolumn{4}{c}{ Generated Words } & \multicolumn{3}{c}{ Unique words in the combined sets } \\
\cline { 2 - 8 } & Happy & Sad & Contempt & Attractiveness & $\begin{array}{l}\text { Happy } \\
\text { and Sad }\end{array}$ & $\begin{array}{l}\text { Happy and } \\
\text { Contempt }\end{array}$ & $\begin{array}{l}\text { Sad and } \\
\text { Contempt }\end{array}$ \\
\hline Study 1 & 2152 & 2156 & 2158 & 2160 & 702 & 815 & 792 \\
Study 2 & 2349 & 2346 & 2315 & 2299 & 639 & 705 & 699 \\
\hline
\end{tabular}

Note. Generated words $=$ number of words that were generated in total by all participants and that had a semantic representation in the semantic space; Unique words in the combined sets = number of words that are unique when combining the two sets.

\section{Inter-rater reliability}

All trained models, except for the attractiveness model grouped according to pictures, were significant in Study 1 (Table S2). The semantic questions approach (using semantic similarity scales as well as semantic predicted scales) yields considerable higher inter-rater reliability compared with rating scales for all dimensions except attractiveness (Table S3). The Krippendorff's alpha values were compared the same way as in the main manuscript. The $t$ tests showed that the semantic measures yielded a significantly higher Krippendorff's alpha compared with the rating scales for all dimensions $(p<.001$; Table S4), except for attractiveness, where rating scales were significantly $(p<.001)$ higher. In addition, employing semantic predictions of ANEW valence applied to the semantic responses also results in significantly higher $(p<.001)$ inter-rater reliability $\left(\operatorname{ICC}_{(2,1)}=.736\right.$; Krippendorff's $\alpha=.71$, $\mathrm{CI}=.69-.73)$. 
Table S2

Pearson's $r$ between Actual and

Predicted Scores from Trained

Models with Subjects or Pictures

as Grouping Variable

\begin{tabular}{lll}
\hline Model & \multicolumn{2}{c}{$\mathbf{r}$} \\
\hline Valence & .89 & .88 \\
Intensity & .74 & .68 \\
Clarity & .77 & .73 \\
Genuineness & .85 & .82 \\
Attractiveness & .36 & $\mathrm{~ns}$ \\
\hline Note. N=119, all correlations were \\
significant at p<.001 unless otherwise \\
specified, ns=not significant. The models \\
were trained to the mean score from the \\
rating scales condition.
\end{tabular}


Table S3

Krippendorff's Alpha $(\alpha)$ and Inter-Class Correlation Coefficient (ICC)

\begin{tabular}{|c|c|c|c|c|c|c|c|c|}
\hline \multirow[t]{4}{*}{ Dimensions } & \multicolumn{6}{|c|}{ Three descriptive words (N: $\alpha=119 ;$ ICC $=116)^{1}$} & \multicolumn{2}{|c|}{ Rating scales (N=148) } \\
\hline & \multicolumn{4}{|c|}{ Trained scales } & \multirow{2}{*}{\multicolumn{2}{|c|}{ Bipolar similarity scales }} & \multirow[b]{3}{*}{$\alpha(95 \%$ CI $)$} & \multirow[b]{3}{*}{ ICC $(2,1)$} \\
\hline & \multicolumn{2}{|c|}{ Participants } & \multicolumn{2}{|c|}{ Pictures } & & & & \\
\hline & $\alpha(95 \% \mathrm{CI})$ & ICC (2,1) & $\alpha(95 \% \mathrm{CI})$ & ICC $(2,1)$ & $\alpha(95 \%$ CI $)$ & ICC $(2,1)$ & & \\
\hline Expression $^{2}$ & $.55(.46-.63)$ & NA & $.50(.41-.59)$ & NA & $\begin{array}{l}.53(.44-.62) \\
.53(.43-.62)^{3}\end{array}$ & NA & $.49(.47-.52)$ & NA \\
\hline Valence & .79(.79-.80) & .803 & $.79(.78-.79)$ & 795 & $.79(.78-.79)$ & 799 & $.38(.31-.45)$ & 394 \\
\hline Intensity & $.65(.64-.66)$ & 661 & $.63(.61-.64)$ & 638 & . 48 (.46-.49) & 494 & $.25(.17-.33)$ & 264 \\
\hline Clarity & $.66(.65-.67)$ & 672 & .63 (.62-.64) & 641 & .64 (.63-.65) & 649 & $.28(.20-.36)$ & 296 \\
\hline Genuineness & $.79(.78-.80)$ & 799 & $.77(.76-.78)$ & .777 & $.62(.60-.63)$ & 636 & $.16(.08-.25)$ & 173 \\
\hline Attractiveness & .08 (.05-.11) & .156 & $.02(-.06-.11)$ & .028 & $.15(.14-.16)$ & 192 & $.33(.26-.40)$ & .374 \\
\hline
\end{tabular}

Note. ${ }^{1} \mathrm{As}$ ICC is sensitive to missing values participants are removed so that in the semantic questions condition these computations are based on 116 participants. ${ }^{2}$ The inter-rater reliability of expressions is based on nominal data; the other dimensions on interval. ${ }^{3}$ unipolar. 
Table S4

Independent T-tests between Krippendorff's Alpha of

Semantic Measures and Rating Scales in Study 1

\begin{tabular}{|c|c|c|c|c|}
\hline \multirow{2}{*}{$\begin{array}{l}\text { Semantic measure } \\
\text { compared with } \\
\text { corresponding } \\
\text { rating scale }\end{array}$} & \multicolumn{2}{|c|}{$\begin{array}{c}\text { M (SD) for } \\
\text { Krippendorff's } \alpha\end{array}$} & \multirow{2}{*}{$\begin{array}{l}\text { Degrees } \\
\text { of } \\
\text { freedoms }\end{array}$} & \multirow[t]{2}{*}{$\mathbf{t}$} \\
\hline & $\begin{array}{l}\text { Semantic } \\
\text { measure }\end{array}$ & $\begin{array}{l}\text { Rating } \\
\text { scale }\end{array}$ & & \\
\hline \multicolumn{5}{|l|}{ Expression } \\
\hline Bipolar-SSS & $.53(.044)$ & $.49(.013)$ & 1176 & 25.2 \\
\hline Unipolar-SSS & $.53(.049)$ & $"$ & 1145 & 20.2 \\
\hline SPS-Participants & $.55(.045)$ & $"$ & 1169 & 36.0 \\
\hline SPS-Pictures & $.50(.046)$ & $"$ & 1164 & 3.9 \\
\hline \multicolumn{5}{|l|}{ Valence } \\
\hline Bipolar-SSS & $.79(.003)$ & $.38(.034)$ & 1015 & 375.8 \\
\hline SPS-Participants & $.79(.003)$ & $"$ & 1016 & 380.7 \\
\hline SPS-Pictures & $.79(.003)$ & $"$ & 1016 & 373.8 \\
\hline Valence-ANEW & $.71(.010)$ & $"$ & 1177 & 294.0 \\
\hline \multicolumn{5}{|l|}{ Intensity } \\
\hline Bipolar-SSS & $.48(.008)$ & $.25(.041)$ & 1082 & 168.0 \\
\hline SPS-Participants & $.65(.006)$ & $"$ & 1041 & 300.6 \\
\hline SPS-Pictures & $.63(.006)$ & $"$ & 1045 & 282.2 \\
\hline \multicolumn{5}{|l|}{ Clarity } \\
\hline Bipolar-SSS & $.64(.004)$ & $.28(.040)$ & 1024 & 277.7 \\
\hline SPS-Participants & $.66(.005)$ & $"$ & 1035 & 294.9 \\
\hline SPS-Pictures & $.63(.005)$ & $"$ & 1034 & 269.6 \\
\hline \multicolumn{5}{|l|}{ Genuineness } \\
\hline Bipolar-SSS & $.62(.006)$ & $.16(.044)$ & 1039 & 323.1 \\
\hline SPS- Participants & $.79(.005)$ & $"$ & 1024 & 446.6 \\
\hline SPS-Pictures & $.77(.005)$ & $"$ & 1025 & 431.9 \\
\hline \multicolumn{5}{|l|}{ Attractiveness } \\
\hline Bipolar-SSS & $.15(.007)$ & $.33(.037)$ & 1064 & -153.8 \\
\hline SPS-Participants & $.08(.017)$ & $"$ & 1397 & -194.7 \\
\hline SPS-Pictures & $.02(.044)$ & $"$ & 1947 & -170.5 \\
\hline
\end{tabular}

Note. All tests were significant at $\mathrm{p}<.001$. For all comparisons Levene's test for equality of variances was significant; hence presented statistical tests are based on equal variance not assumed. SSS = Semantic Similarity Scale; SPS-Participants = Semantic Predicted Scale with Participants as grouping variable; SPS-Pictures = Semantic Predicted Sale with Pictures as grouping variable. $\mathrm{N}=1000$ in each comparison group.

Methods Used for Fine-Tuning the Analyses. Different ways of using and constructing the word norms were evaluated and applied in the analyses. First, the similarity scales of the different norms were normalized to z-values (this was not done for the rating scale scores, as the z-transformation did not improve their categorization). Second, as described in the construction of norms, the targeted word (used in the question generating the word norm) was added (i.e., $f(\max )+1)$, as this was found to increase the validity of the method. That is, when 
asking participants to describe an imagined happy face they rarely answer happy (probably because this goes without saying). An important aspect, however, is that we found that simply using the targeted word as a norm did not yield results as valid as when including the words generated by the participants. Cleaning the word norms so that they only included words that had been generated at least two times did not yield higher validity and was thus not applied. Third, the normalization, as described in the Methodological and Statistical Approach section, was employed to correct for artifacts relating to frequently occurring words. This normalization yielded somewhat more valid results. We furthermore believe that this normalization is particularly important for the validity of the method when the semantic responses differ more in length than in the current studies.

\section{Discussion of Study 1}

Except for attractiveness, the semantic questions approach yields considerably higher validity as indicated by significantly higher levels of correct categorizations of expressions, as well as higher inter-rater reliability compared with the rating scales. However, this comparison may be regarded as unfair due to the fact that there were nine possible categorization alternatives in the rating scales condition, and only three possible outcomes in the semantic questions condition. That is, the word responses were categorized into one of three categories, resulting in a $1 / 3$ chance of being correct if categorizing at random; as there were nine alternatives in the rating scale condition, there is a 1/9 chance through random categorization. This concern is addressed in Study 2 by controlling for the number of categorization alternatives. Thus, three rating scales were used for rating the degree of happiness, sadness and contempt for every expression, which thus also result in a $1 / 3$ chance. Although attractiveness is not a part of the main focus of this study, we clarified the semantic question for Study 2 by emphasizing that participants should describe how "unattractive or attractive" they perceive the person in the picture. 


\section{Supplementary Material for Study 2}

Table S5

Pearson's r between Empirical and

Predicted Scores from Trained Models

\begin{tabular}{lll}
\hline \multirow{2}{*}{ Dimension } & \multicolumn{2}{c}{$\mathbf{r}$} \\
\cline { 2 - 3 } Happy & Participants & Pictures \\
Sad & .91 & .90 \\
Contemptuous & .83 & .80 \\
Valence & .91 & .76 \\
Intensity & .76 & .90 \\
Clarity & .79 & .69 \\
Genuineness & .85 & .75 \\
Arousal & .85 & .84 \\
Attractiveness & .53 & .85 \\
\hline
\end{tabular}

Note. $\mathrm{N}=133$, all correlations were significant at $\mathrm{p}<.001$ unless otherwise specified, ns=not significant. The models were trained to the mean scores from the rating scales condition. The $10 \%$ leave-out procedure was either grouped based on Participants or Pictures. 
Table S6

Independent T-tests between Krippendorff's Alpha of Semantic Measures and Rating Scales in Study 2

\begin{tabular}{|c|c|c|c|c|}
\hline \multirow{2}{*}{$\begin{array}{l}\text { Semantic } \\
\text { measure } \\
\text { compared with } \\
\text { corresponding } \\
\text { rating scale }\end{array}$} & \multicolumn{2}{|c|}{$\begin{array}{c}\text { M (SD) for } \\
\text { Krippendorff's } \alpha\end{array}$} & \multirow{2}{*}{$\begin{array}{l}\text { Degrees } \\
\text { of } \\
\text { freedoms }\end{array}$} & \multirow[t]{2}{*}{$\mathbf{t}$} \\
\hline & $\begin{array}{l}\text { Semantic } \\
\text { measures }\end{array}$ & $\begin{array}{l}\text { Rating } \\
\text { scale }\end{array}$ & & \\
\hline \multicolumn{5}{|l|}{ Expression } \\
\hline Bipolar-SSS & $.57(.045)$ & $.48(.019)$ & 1357.26 & 57.1 \\
\hline Unipolar-SSS & $.58(.045)$ & $"$ & 1350.33 & 64.3 \\
\hline SPS-Participants & $.61(.043)$ & $"$ & 1387.13 & 85.6 \\
\hline SPS-Pictures & $.53(.046)$ & $"$ & 1337.06 & 33.0 \\
\hline \multicolumn{5}{|l|}{ Valence } \\
\hline Bipolar-SSS & $.82(.003)$ & $.31(.037)$ & 1008.78 & 440.0 \\
\hline SPS- Participants & $.81(.005)$ & $"$ & 1030.76 & 432.3 \\
\hline SPS- Pictures & $.81(.004)$ & $"$ & 1026.34 & 426.1 \\
\hline V-ANEW & $.74(.010)$ & $"$ & 1152.76 & 355.5 \\
\hline \multicolumn{5}{|l|}{ Arousal } \\
\hline Bipolar-SSS & $.72(.004)$ & $.13(.044)$ & 1017.59 & 422.6 \\
\hline SPS- Participants & $.77(.008)$ & " & 1071.77 & 451.5 \\
\hline SPS- Pictures & $.77(.008)$ & $"$ & 1058.03 & 454.6 \\
\hline \multicolumn{5}{|l|}{ Intensity } \\
\hline Bipolar-SSS & $.53(.008)$ & $.24(.044)$ & 1057.25 & 206.4 \\
\hline SPS- Participants & $.64(.011)$ & $"$ & 1130.50 & 277.7 \\
\hline $\begin{array}{l}\text { SPS- Pictures } \\
\text { Clarity }\end{array}$ & $.62(.011)$ & $"$ & 1118.35 & 263.4 \\
\hline Bipolar-SSS & $.67(.004)$ & $.24(.044)$ & 1018.42 & 304.8 \\
\hline SPS- Participants & $.66(.008)$ & $"$ & 1071.42 & 288.8 \\
\hline SPS- Pictures & $.64(.008)$ & $"$ & 1065.45 & 274.3 \\
\hline Genuineness & & & & \\
\hline Bipolar-SSS & $.68(.006)$ & $.17(.045)$ & 1031.87 & 353.6 \\
\hline SPS- Participants & $.77(.010)$ & $"$ & 1092.70 & 411.6 \\
\hline $\begin{array}{l}\text { SPS- Pictures } \\
\text { Attractiveness }\end{array}$ & $.77(.010)$ & $"$ & 1091.90 & 411.9 \\
\hline Bipolar-SSS & $.21(.006)$ & $.31(.039)$ & 1052.86 & -83.8 \\
\hline SPS- Participants & $.21(.018)$ & " & 1386.427 & -78.0 \\
\hline SPS- Pictures & $.01(.047)$ & $"$ & 1933.65 & -158.5 \\
\hline
\end{tabular}

Note. All tests were significant at $\mathrm{p}<.001$. For all comparisons Levene's test for equality of variances was significant; hence presented statistical tests are based on equal variance not assumed. SSS = Semantic Similarity Scale; SPS-Participants = Semantic Predicted Scale with Participants as grouping variable; SPS-Pictures $=$ Semantic Predicted Sale with Pictures as grouping variable. $\mathrm{N}=$ 1000 in each comparison group. 


\section{Supplementary Material for Reports regarding Subjective States}

\begin{tabular}{|c|c|c|c|}
\hline \multirow{2}{*}{ Study: condition } & \multicolumn{2}{|c|}{ Generated words } & \multirow{2}{*}{$\begin{array}{l}\text { Unique words } \\
\text { in the } \\
\text { combined sets } \\
\begin{array}{l}\text { Harmony and } \\
\text { Satisfaction }\end{array}\end{array}$} \\
\hline & Harmony & Satisfaction & \\
\hline Study 3: words & 872 & 866 & 792 \\
\hline Study 3: texts & 5998 & 6620 & 1436 \\
\hline Study 4: words & 2969 & 2993 & 1682 \\
\hline Study 4: texts & 24354 & 26671 & 3314 \\
\hline Study 5: words & 2875 & 2917 & 1845 \\
\hline Study 5: texts & 23120 & 25131 & 3356 \\
\hline Study: $3-5$ texts & 53472 & 58422 & 4885 \\
\hline Study 6: words & 1886 & 1913 & 1301 \\
\hline Study 7: 1 word & 364 & 360 & 305 \\
\hline Study 7: 3 words & 1067 & 1060 & 723 \\
\hline Study 7: 5 words & 1296 & 1284 & 896 \\
\hline Study 9 T1: words & 8682 & 8621 & 3063 \\
\hline \multirow[t]{2}{*}{ Study 9 T2: words } & 4758 & 4750 & 2136 \\
\hline & Worry & Depression & $\begin{array}{l}\text { Worry and } \\
\text { Depression }\end{array}$ \\
\hline Study 8: words & 3926 & 3958 & 1832 \\
\hline Study 9 T2: words & 4754 & 4723 & 2060 \\
\hline $\begin{array}{l}\text { Note. Generated } \\
\text { generated in total } \\
\text { representation in } \\
\text { combined sets } \\
\text { combining the tw }\end{array}$ & $\begin{array}{l}\text { humber of } \\
\text { sets. }\end{array}$ & $\begin{array}{l}\text { mber of wor } \\
\text { ants and that } 1 \\
\text { pace; Unique } \\
\text { ords that are }\end{array}$ & $\begin{array}{l}\text { ls that were } \\
\text { ad a semantic } \\
\text { words in the } \\
\text { unique when }\end{array}$ \\
\hline
\end{tabular}

\section{Semantic Questions with Descriptive Text Responses}

\section{Predicting Rating Scale Scores from Semantic Text Responses}

Training the text responses for harmony in life and satisfaction with life to the rating scales yield significant correlations (Table S8). Harmony-texts consistently predict HILS-scores more strongly than SWLS-scores; whilst this is not always the case for satisfaction texts, which predict both HILS-scores and SWLS-scores to a similar degree. Even though the correlations between predicted and actual scores are lower $(r=.49$ for harmony text and HILS and $r=.44$ for 
satisfaction text and SWLS in Study 3-5) as compared with the descriptive words approach, the correlations based on covarying the semantic predicted ANEW valence scales tend to be as strong for text-based responses as for the descriptive words responses. The semantic texts responses can also be trained to predict rating scales of depression, anxiety and stress (Table S8). Although, these correlations tend to be smaller than for the rating scales of harmony and satisfaction, which the current semantic questions aim to measure. In sum, these correlations support that the semantic questions approach with text as response format can measure targeted psychological constructs.

Table S8

Training Semantic Text Responses with Numeric Rating Scales Show Validity

\begin{tabular}{|c|c|c|c|c|c|}
\hline \multirow[t]{2}{*}{ Correlation items } & & \multicolumn{4}{|c|}{ Pearson's r } \\
\hline & & $\begin{array}{l}\text { Study } 3 \\
(\mathrm{~N}=92)\end{array}$ & $\begin{array}{l}\text { Study } 4 \\
(\mathrm{~N}=303)\end{array}$ & $\begin{array}{l}\text { Study } 5 \\
(\mathrm{~N}=294)\end{array}$ & $\begin{array}{l}\text { Study 3-5 } \\
(N=689)\end{array}$ \\
\hline \multirow[t]{2}{*}{ Harmony texts } & HILS & $.32 * *$ & $.41 * * *$ & $.41 * * *$ & $.49 * * *$ \\
\hline & SWLS & ns & $.20 * * *$ & $.19 * *$ & $.41 * * *$ \\
\hline \multirow{2}{*}{$\begin{array}{l}\text { Harmony texts (SP Valence } \\
\text { scale as covariate) }\end{array}$} & HILS & ns & $.28 * * *$ & $.22 * * *$ & $.29 * * *$ \\
\hline & SWLS & ns & ns & ns & $.20 * * *$ \\
\hline \multirow[t]{2}{*}{ Satisfaction texts } & SWLS & $.19 *$ & $.42 * * *$ & $.38 * * *$ & $.44 * * *$ \\
\hline & HILS & $.38 * * *$ & $.49 * * *$ & $.38 * * *$ & $.48 * * *$ \\
\hline \multirow{2}{*}{$\begin{array}{l}\text { Satisfaction texts (SP Valence } \\
\text { scale as covariate) }\end{array}$} & SWLS & $\mathrm{ns}$ & $.35 * * *$ & $.15 * *$ & $.29 * * *$ \\
\hline & HILS & $.25 * *$ & $.38 * * *$ & $.16^{* *}$ & $.30 * * *$ \\
\hline HILS & SWLS & $.80 * * *$ & $.84 * * *$ & $.88 * * *$ & $.85^{* * *}$ \\
\hline
\end{tabular}

Note. $*=\mathrm{p}<.05 ; * *=\mathrm{p}<.01 ; * * *=\mathrm{p}<.001 ; \mathrm{ns}=$ not significant. HILS = Harmony in Life Scale; SWLS=Satisfaction with Life Scale; SP=Semantic Predicted. 
Table S9

Pearson's $r$ Between Rating Scales and Trained Semantic

Predictions from Generated Words and Text in Study 5

\begin{tabular}{|c|c|c|c|}
\hline & Depression & Anxiety & Stress \\
\hline HILS & $-.69 * * *$ & $-.43 * * *$ & $-.57 * * *$ \\
\hline SWLS & $-.64 * * *$ & $-.36 * * *$ & $-.50 * * *$ \\
\hline Depression & - & $.76^{* * *}$ & $.78 * * *$ \\
\hline Anxiety & & - & $.80 * * *$ \\
\hline Stress & & & - \\
\hline \multicolumn{4}{|l|}{ Words/Text responses } \\
\hline Harmony words & $.47 * * *$ & $.36 * * *$ & $.34 * * *$ \\
\hline Covary SP Valence scale & $.20 * * *$ & $.28 * * *$ & $.13 *$ \\
\hline Satisfaction words & $.54 * * *$ & $.32 * * *$ & $.34 * * *$ \\
\hline Covary SP Valence scale & $.19 * * *$ & $.21 * * *$ & $.10 *$ \\
\hline Harmony text & $.28 * * *$ & ns & ns \\
\hline Co-vary SP Valence scale & $.15^{* *}$ & ns & ns \\
\hline Satisfaction text & $.30 * * *$ & $.23 * * *$ & $.25 * * *$ \\
\hline Co-vary SP Valence scale & $.17 * *$ & $.13 *$ & $.13 *$ \\
\hline
\end{tabular}

The performance functions for the analyses of text-responses for studies 3-5 combined are presented in Table S10-S12. In accordance with descriptive words responses, significant correlations appear when the sample size reaches 64 participants (Table S10). The first word accounts for a large part of the predictive power (Table S11). Including more than 128 words do not increase the correlation very much (Table S12). However, the mean amount of words written for each participant were 78 for harmony and 85 for satisfaction; so, if participants had written longer texts this might further have increased the correlations. Lastly, including the entire sample yields the highest correlations for harmony words; hence it might be possible to receive even higher correlations in larger samples. 
Table S10

Correlations between

Predicted and Actual Rating

Scale Scores as a Function

of Number of Participants on

Text-Responses

Function of $\mathbf{N}$ participants

\begin{tabular}{lll} 
& \multicolumn{2}{c}{ Pearson's r } \\
\hline N-ps & Ht: HILS & St: SWLS \\
\hline $\mathbf{8}$ & $\mathrm{ns}$ & $\mathrm{ns}$ \\
$\mathbf{1 6}$ & $\mathrm{ns}$ & $\mathrm{ns}$ \\
$\mathbf{3 2}$ & $\mathrm{ns}$ & $\mathrm{ns}$ \\
$\mathbf{6 4}$ & $.27 *$ & $.29 * *$ \\
$\mathbf{1 2 7}$ & $.27 * *$ & $.26 * *$ \\
$\mathbf{2 5 5}$ & $.33 * * *$ & $.37 * * *$ \\
$\mathbf{5 1 0}$ & $.44 * * *$ & $.44 * * *$ \\
$\mathbf{6 8 9}$ & $.49 * * *$ & $.44 * * *$ \\
\hline Note. $\mathrm{N}=689 ; *=\mathrm{p}<.05 ; * *=\mathrm{p}<.01 ;$
\end{tabular}

Note. $\mathrm{N}=689 ; *=\mathrm{p}<.05 ; * *=\mathrm{p}<.01$; $* * *=\mathrm{p}<.001 ; \mathrm{ns}=$ not significant; $\mathrm{N}$ ps $=$ Number of participants; $\mathrm{Ht}=$ Harmony text; St = Satisfaction text; HILS = Harmony in Life Scale; SWLS = Satisfaction with Life Scale. 
Table S11

Correlations

between

Predicted and Actual Rating

Scale Scores as a Function of

Position of Words on Text-

Responses

Function of word at serial position $\mathbf{N}$

Pearson's $r$

\begin{tabular}{lll}
\hline $\begin{array}{l}\text { Word } \\
\text { position }\end{array}$ & Ht: HILS & St: SWLS \\
\hline $\mathbf{1}^{\text {st }}$ & $.24 * * *$ & $.10^{* *}$ \\
$\mathbf{2}^{\text {nd }}$ & $.12^{* *}$ & $\mathrm{~ns}$ \\
$\mathbf{3}^{\text {rd }}$ & $.22^{* * *}$ & $.29 * * *$ \\
$\mathbf{4}^{\text {th }}$ & $.18^{* * *}$ & $\mathrm{~ns}$ \\
$\mathbf{5}^{\text {th }}$ & $.09 *$ & $.19^{* *}$ \\
$\mathbf{6}^{\text {th }}$ & $\mathrm{ns}$ & $\mathrm{ns}$ \\
$\mathbf{7}^{\text {th }}$ & $.11^{* *}$ & $\mathrm{~ns}$ \\
$\mathbf{8}^{\text {th }}$ & $\mathrm{ns}$ & $.11^{* *}$ \\
$\mathbf{9}^{\text {th }}$ & $\mathrm{ns}$ & $\mathrm{ns}$ \\
$\mathbf{1 0}$ & $\mathrm{ns}$ & $\mathrm{ns}$ \\
\hline Note. N= 689; & $* \mathrm{p}<.05 ; * *=\mathrm{p}<.01 ;$ \\
$*^{\text {th }}$ & $\mathrm{n}<.001 ; \mathrm{ns}=$ not significant. Ht \\
$=$ Harmony text; St = Satisfaction \\
text; HILS = Harmony in Life \\
Scale; SWLS = Satisfaction with \\
Life Scale.
\end{tabular}


Table S12

Correlations between

Predicted and Actual Rating

Scale Scores as a Function of

Number of Words in the Text-

Responses

\begin{tabular}{|c|c|c|}
\hline \multicolumn{3}{|c|}{$\begin{array}{c}\begin{array}{c}\text { Function of N-first-words } \\
\text { Pearson's r }\end{array} \\
\end{array}$} \\
\hline $\begin{array}{l}\mathrm{N}- \\
\text { words }\end{array}$ & Ht: HILS & St: SWLS \\
\hline 1 & $.24 * * *$ & $.10^{* *}$ \\
\hline 2 & $.23 * * *$ & $.14 * * *$ \\
\hline 4 & $.42 * * *$ & $.37 * * *$ \\
\hline 8 & $.34 * * *$ & $.35 * * *$ \\
\hline 16 & $.33 * * *$ & $.37 * * *$ \\
\hline 32 & $.28 * * *$ & $.30 * * *$ \\
\hline 64 & $.40 * * *$ & $.36 * * *$ \\
\hline 128 & $.48 * * *$ & $.42 * * *$ \\
\hline 256 & $.49 * * *$ & $.43 * * *$ \\
\hline 512 & $.49 * * *$ & $.44 * * *$ \\
\hline \multicolumn{3}{|c|}{$\begin{array}{l}\text { Note. } \mathrm{N}=689 ; \quad *=\mathrm{p}<.05 ; \quad * *=\mathrm{p}<.01 \\
* * *=\mathrm{p}<.001 ; \mathrm{Ht}=\mathrm{Harmony} \text { text; } \mathrm{St}= \\
\text { Satisfaction text; HILS = Harmony in } \\
\text { Life Scale; SWLS = Satisfaction with } \\
\text { Life Scale. }\end{array}$} \\
\hline
\end{tabular}

\section{Word-Norms on Text-Responses}

The correlations with the bipolar semantic similarity scales yield significant correlations; although, they are approximately half the size compared with those based on descriptive words rather than text based responses. The correlations between the text based semantic similarity scales, trained semantic scales, and rating scales are presented in Table S13. The bipolar semantic similarity scales for harmony-disharmony and satisfaction-dissatisfaction, as well as the semantic predicted ANEW valence scales yield the highest correlations with the rating scales. The unipolar semantic similarity scales for satisfaction and harmony do not correlate significantly with the rating scales; meanwhile the semantic similarity scales for disharmony and dissatisfaction correlate significantly with respective rating scale. Hence, it is possible to measure participants' degree of harmony and satisfaction using the text-responses from the semantic questions. 
Table S13

Pearson's r among Semantic Scales and Numerical Rating Scales for Text Data in Study 3-5

\begin{tabular}{|c|c|c|c|c|c|c|c|c|c|}
\hline & 1 & 2 & 3 & 4 & 5 & 6 & 7 & 8 & 9 \\
\hline \multicolumn{10}{|l|}{ 1. HILS } \\
\hline 2. SWLS & $.85 * * *$ & & & & & & & & \\
\hline 3. Ht: Valence & $.46 * * *$ & $.39 * * *$ & & & & & & & \\
\hline 4. St: Valence & $.41 * * *$ & $.41 * * *$ & $.47 * * *$ & & & & & & \\
\hline 5. Ht: H-unipolar & ns & ns & ns & ns & & & & & \\
\hline 6. St: S-unipolar & ns & ns & ns & ns & $.42 * * *$ & & & & \\
\hline 7. Ht: Dh-unipolar & $-.13 * * *$ & $-.11 * *$ & $-.10 * *$ & ns & $.98 * * *$ & $.43 * * *$ & & & \\
\hline 8. St: Ds-unipolar & ns & $-.08^{*}$ & $-.08^{*}$ & ns & $.42 * * *$ & $.99 * * *$ & $.45 * * *$ & & \\
\hline 9. Ht: bipolar & $.37 * * *$ & $.30 * * *$ & $.68 * * *$ & $.32 * * *$ & $-.30 * * *$ & $-.22 * * *$ & $-.48 * * *$ & $-.28 * * *$ & \\
\hline 10. St: bipolar & $.42 * * *$ & $.44 * * *$ & $.40 * * *$ & $.76 * * *$ & $-.12 * *$ & $-.14 * * *$ & $-.20 * * *$ & $-.29 * * *$ & $.42 * * *$ \\
\hline
\end{tabular}

Note. $\mathrm{N}=689 ; *=\mathrm{p}<.05 ; * *=\mathrm{p}<.01 ; * * *=\mathrm{p}<.001$. HILS=Harmony in Life Scale; SWLS=Satisfaction with Life Scale; $\mathrm{Ht}=$ Harmony text; $\mathrm{St}=$ Satisfaction text; H=Harmony; S=Satisfaction; Dh=Disharmony; Ds=Dissatisfaction; Valence=Semantic Predicted Valence Scales; Bipolar=Bipolar Semantic Similarity Scale; Unipolar=Unipolar Semantic Similarity Scale. 


\section{Describing and Differentiating Constructs}

Semantic t-tests reveal that the texts generated in relation to harmony in life and satisfaction with life significantly differ from each other with large effect sizes (Table S14). Further, Figure S1 a-i demonstrates that satisfaction and harmony conceptually differ; where satisfaction tap into self-fulfilment and independence (e.g., see job and $a m$ as in "I am") and harmony to balance and interdependence (e.g., see balance and between). Importantly though, the keyword plots for the text-responses differ from the descriptive words figures as these reveal more function words such as pronouns, particles, prepositions etc. This links to Pennebaker and colleagues' findings that different usage of function words relate to mental health (Pennebaker et al., 2003). Hence, text-responses contribute to some extent with important information beyond the descriptive words approach.

The text-based figures (as for the descriptive words), also suggest that semantic similarity scales measure the constructs more clearly than rating scales: that is, when covarying the semantic similarity scales of harmony and satisfaction with each other more words stay significant (Figure S1e-f; 40 words for both figures) as compared to when the harmony and satisfaction rating scales are covaried with each other (Figure S1g-h; 2 and 0 words, respectively).

In summary, text responses can be used to measure, differentiate and describe harmony in life and satisfaction with life. Text-responses, as compared with descriptive words, yield lower correlations to respective rating scales for both semantic similarity and semantic predicted scales. However, this response format is important as it involves writing full sentences rather than only descriptive words, which can, for example, highlight function words. 
Table S14

Semantic T-Tests between Harmony in life and Satisfaction with life Text Responses Differ

Significantly

\begin{tabular}{lllll}
\hline Study & $\begin{array}{l}\text { Study 3 } \\
(\mathbf{N = 9 2 )}\end{array}$ & $\begin{array}{l}\text { Study 4 } \\
(\mathbf{N = 3 0 3 )})\end{array}$ & $\begin{array}{l}\text { Study 5 } \\
(\mathbf{N = 2 9 4})\end{array}$ & $\begin{array}{l}\text { Study 3-5 } \\
(\mathbf{N}=689)\end{array}$ \\
\hline t-value & 9.21 & 23.72 & 22.22 & 34.38 \\
Cohen's d & .96 & 1.36 & 1.30 & 1.31 \\
\hline
\end{tabular}

Note. All differences were significant at $\mathrm{p}<.001$

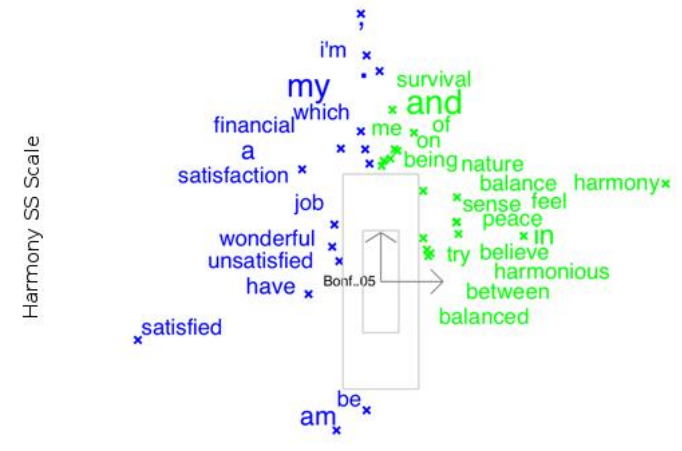

Satisfaction texts vs. Harmony texts

a

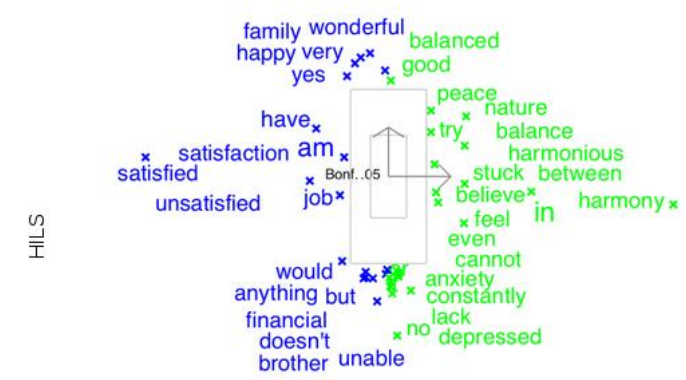

net

Satisfaction texts vs. Harmony texts

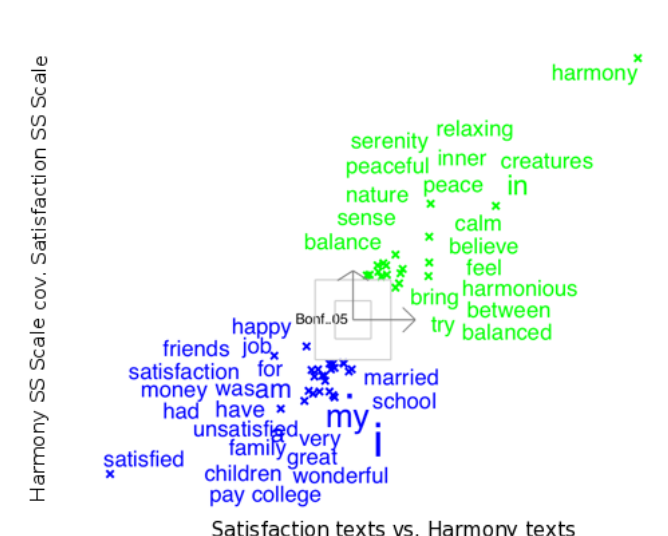

e

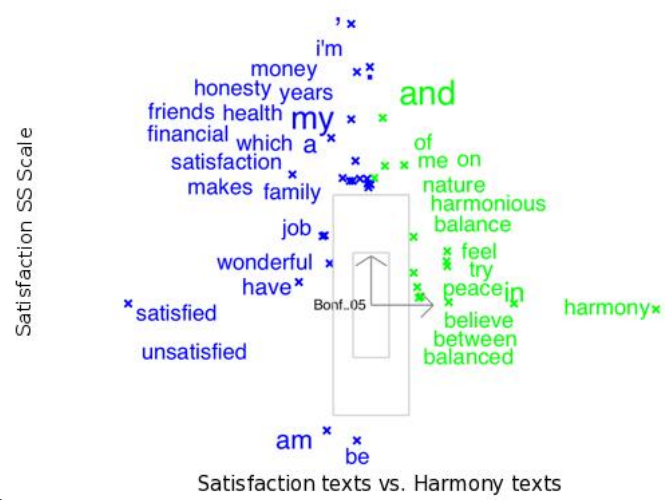

b

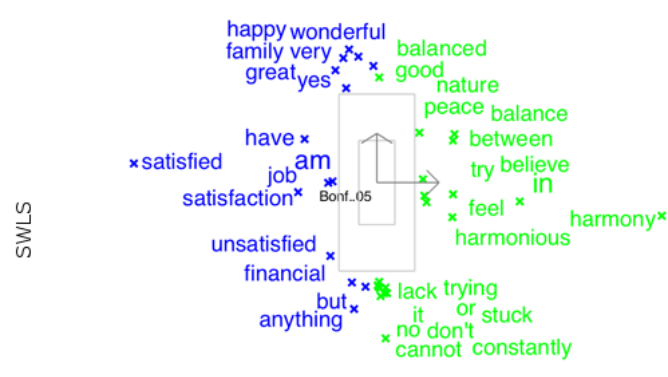

not

d

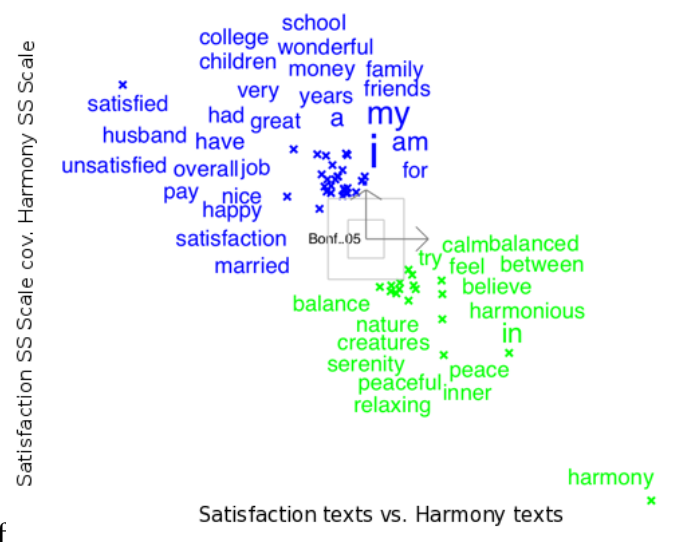



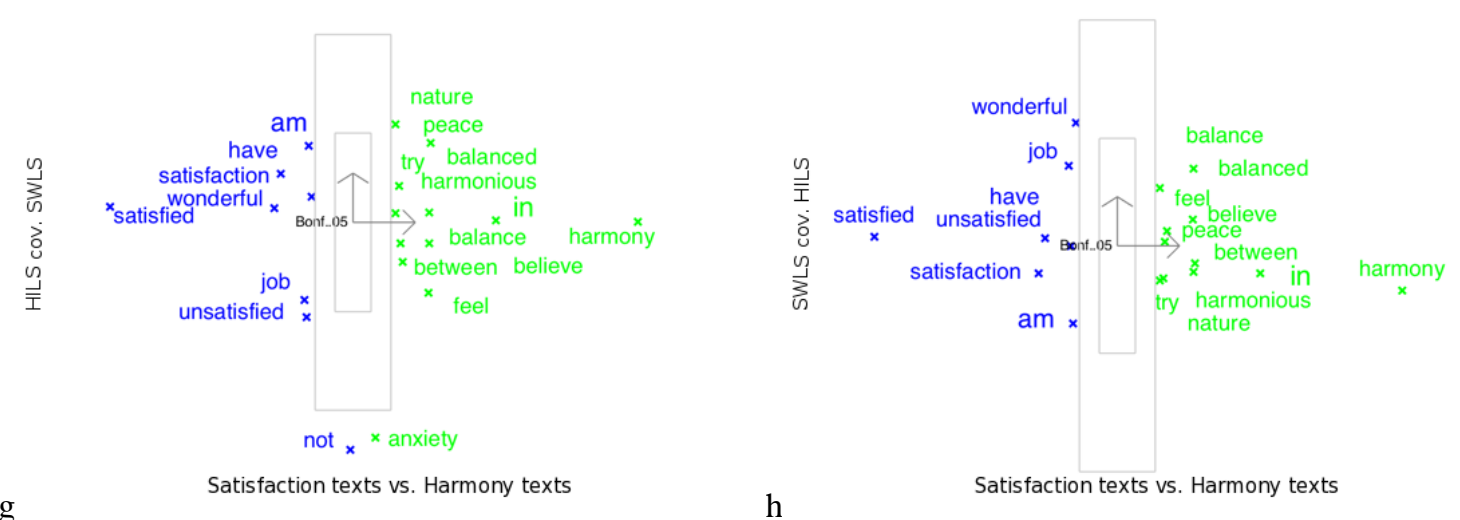

g

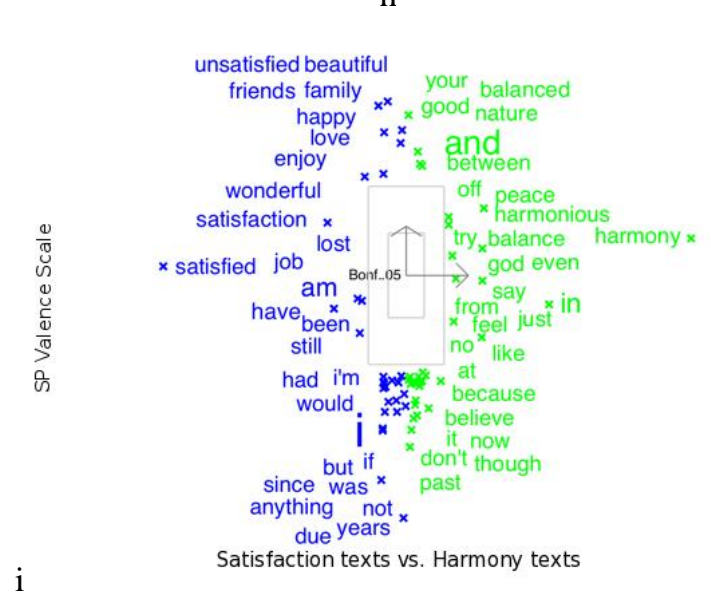

Figure S1 a-i. Semantic measures based on text responses describe constructs well, whilst highlighting more function words as compared with descriptive word responses. Semantic measures (e, f) differentiate between constructs better than rating scales $(\mathrm{g}, \mathrm{h})$; as can be seen most clearly when comparing e with $\mathrm{g}$ and $\mathrm{f}$ with $\mathrm{h}$. On the $\mathrm{x}$-axis, words are plotted according to the $\chi$-values from a Chisquare test, with a Bonferroni correction for multiple comparisons (Bonf.=Bonferroni line where $\chi$ $=4.13, .05$ indicates the uncorrected $\mathrm{p}$-value line, where $\chi=1.96)$. On the $y$-axis, words are plotted according to Pearson's $\mathrm{r}(\mathrm{r}=.11$ at the Bonferroni line, and $\mathrm{r}=.05$ at the .05 uncorrected $\mathrm{p}$-value line). More frequent words are plotted with a larger font with fixed lower and upper limits. The x-axes represent $\chi$-values associated with words generated in relation to satisfaction (blue/left) versus harmony (green/right). The y-axes show significant words related to Semantic Similarity (SS) scales, Semantic Predicted (SP) scales or rating scales; HILS=Harmony in Life Scale; SWLS=Satisfaction with Life Scale; cov.=covariate. $N=689$. 